\title{
THE REACTION-DIFFUSION MASTER EQUATION AS AN ASYMPTOTIC APPROXIMATION OF DIFFUSION TO A SMALL TARGET
}

\author{
SAMUEL A. ISAACSON*
}

\begin{abstract}
The reaction-diffusion master equation (RDME) has recently been used as a model for biological systems in which both noise in the chemical reaction process and diffusion in space of the reacting molecules is important. In the RDME space is partitioned by a mesh into a collection of voxels. There is an unanswered question as to how solutions depend on the mesh spacing. To have confidence in using the RDME to draw conclusions about biological systems, we would like to know that it approximates a reasonable physical model for appropriately chosen mesh spacings. This issue is investigated by studying the dependence on mesh spacing of solutions to the RDME in $\mathbb{R}^{3}$ for the bimolecular reaction $\mathrm{A}+\mathrm{B} \rightarrow \varnothing$, with one molecule of species $\mathrm{A}$ and one molecule of species $\mathrm{B}$ present initially. We prove that in the continuum limit the molecules never react and simply diffuse relative to each other. Nevertheless, we show that the RDME with non-zero lattice spacing yields an asymptotic approximation to a specific spatially-continuous diffusion limited reaction (SCDLR) model. We demonstrate that for realistic biological parameters it is possible to find mesh spacings such that the relative error between asymptotic approximations to the solutions of the RDME and the SCDLR models is less than one percent.
\end{abstract}

1. Introduction. Noise in the chemical reaction process can play an important role in the dynamics of biochemical systems. In the field of molecular cell biology, this has been convincingly demonstrated both experimentally and through mathematical modeling. The pioneering work of Arkin and McAdams [7] has been followed by numerous studies showing that not only must biological cells compensate for noisy biochemical gene/signaling networks [12, 31, 40,37], but they may also take advantage of the inherent stochasticity in the chemical reaction process $[8,44,33]$.

Until recently, stochastic mathematical models of biochemical reactions within biological cells were primarily non-spatial, treating the cell as a well-mixed volume, or perhaps as several well-mixed compartments (i.e. cytosol, nucleus, ER,...). Biological cells contain incredibly complex spatial environments, comprised of numerous organelles, irregular membrane structures, fibrous actin networks, long directed microtubule bundles, and many other geometrically complex structures. While few authors have modeled the effects of these structures on the dynamics of chemical reactions within biological cells, several have recently begun to investigate what effect the spatially distributed nature of the cell has on biochemical signaling networks $[42,2,38,17,47]$. Deterministic reaction-diffusion partial differential equation models are well-established for modeling biochemical systems in which reactant species are present in sufficiently high concentrations, however, there is not yet a standard model for systems in which noise in the chemical reaction process is thought to be important. Three different $[6,28,15,48]$, but related, mathematical models have recently been used for representing stochastic reaction-diffusion systems in biological cells $[2,38,17,47]$.

In both the methods of [6] and [48] molecules are modeled as points undergoing spatially-continuous Brownian motion, with bimolecular chemical reactions occurring instantly when the molecules pass within specified reaction-radii. We subsequently refer to this model, proposed by Smoluchowski [43], as a spatially-continuous diffusion limited reaction (SCDLR). [6] and [48] differ in their numerical simulation algorithms,

* Department of Mathematics, University of Utah, 155 S. 1400 E., Room 233, Salt Lake City, UT 84112 (isaacson@math.utah.edu) 
but both involve approximations that remain spatially continuous while introducing time discretizations. In contrast to both these methods, the reaction-diffusion master equation (RDME) model used in [15] and [28] discretizes space, approximating the diffusion of molecules as a continuous time random walk on a lattice, with bimolecular reactions occurring with a fixed probability per unit time for molecules within the same voxel. Exact realizations of the reaction-diffusion master equation can be created using the Gillespie Method [21]. The method of [28] shows how to modify the diffusive jump rates of the standard reaction-diffusion master equation approach to account for complex spatial geometries.

While several authors have recently used the reaction-diffusion master equation to study biological systems, see for example [17] and [11], there is still an unanswered question as to whether this spatially-discrete model approximates any underlying physical model for appropriately chosen mesh sizes. (Note that [11] uses an approximate simulation algorithm instead of the exact Gillespie method approach mentioned above). In particular, the main justification for the use and accuracy of the reaction diffusion master equation appears to be the physical separation of timescales argument given in Section 1.1.2. This argument suggests that the reaction-diffusion master equation is only physically valid for mesh sizes that are neither too large or too small, and gives no hint as to an underlying spatially continuous model that is approximated by the reaction-diffusion master equation.

Our purpose herein is to investigate the dependence of the reaction-diffusion master equation on mesh spacing. We begin by answering the question of what happens in the continuum limit that the mesh spacing approaches zero. To this end, we prove in Section 2.1 that for two molecules that can undergo the bimolecular reaction $\mathrm{A}+\mathrm{B} \rightarrow \varnothing$, as the mesh spacing approaches zero the molecules never react and simply diffuse relative to each other. This rigorous result appears to contradict the naive formal continuum limit,

$$
D \Delta-k \delta(\boldsymbol{x}),
$$

that one obtains for the generator of the dynamics (2.7). The apparent contradiction arises from the subtlety of giving a rigorous mathematical definition to the operator (1.1). In the context of quantum mechanical scattering in $\mathbb{R}^{3}$ an equivalent operator, with the reaction term called a pseudo-potential, has been introduced formally by Fermi [19] and elaborated on by Huang and Yang [24]. A rigorous mathematical definition of (1.1) was first given by Berezin and Faddeev [10] and more recently by Albeverio et al. [3]. An important point in the work of [3] is that a one-parameter family of self-adjoint operators, $\Delta+\alpha \delta(\boldsymbol{x})$ may be defined in $\mathbb{R}^{3}$ corresponding to an extension of the standard Laplacian from $\mathbb{R}^{3} \backslash\{(0,0,0)\}$ to $\mathbb{R}^{3}$. The results of Section 2.1 imply that the standard scaling of the bimolecular reaction rate used in the RDME leads the solution of the RDME to converge to the $\alpha=0$ operator, i.e. the Laplacian on $\mathbb{R}^{3}$. To obtain an operator (1.1) corresponding to the formal continuum limit that differs from the Laplacian, one would need to appropriately renormalize the bimolecular reaction rate and/or extend the reaction operator to couple in neighboring voxels.

We next investigate what the reaction-diffusion master equation approximates for mesh spacings that are neither too large or too small. The operator (1.1) arises in Quantum Mechanics to give local potentials whose scattering approximates that of a hard sphere of a fixed radius. Here, the dynamics (2.7) generated by a physically appropriate, mathematically rigorous definition of (1.1) provides an asymptotic approximation to the solution of the SCDLR model. This motivates Section 2.2, where 
we show that for the mesh spacing larger than an appropriately chosen "reactionradius," defined by the relative diffusion constant and bimolecular reaction rate of the species, the reaction-diffusion master equation is an asymptotic approximation to the SCDLR model $[43,29]$. We derive, for the special case of two molecules that can undergo the bimolecular reaction $\mathrm{A}+\mathrm{B} \rightarrow \varnothing$, asymptotic expansions in the reactionradius of the solutions to both the RDME and the SCDLR model in Subsections 2.2.1 and 2.2.2 respectively. In Subsection 2.2.3 we prove that the zero'th and first order terms in the expansion of the reaction-diffusion master equation converge to the corresponding terms of the SCDLR model, while the second order term diverges. Moreover, we examine the numerical error between the expansion of the reaction-diffusion master equation, truncated after the second order term, and the asymptotic expansion of the SCDLR model, also truncated after the second order term. It is shown that for biologically relevant values of the reaction-radius, the relative error between the two truncated expansions can be reduced below one percent with appropriately chosen mesh widths. This suggests that for biologically relevant parameter regimes and wellchosen mesh spacings, the reaction-diffusion master equation might provide a useful approximation to the spatially-continuous diffusion limited reaction model.

The model problem studied in Section 2 is chosen for ease of mathematical analysis. We believe that our results should be extendable to the general reaction-diffusion master equation formulation presented in Section 1.1 for chemical systems with arbitrary zero'th, first, and second order chemical reactions. Note, for a general chemical system the reaction-diffusion master equation is a, possibly infinite, coupled system of ODEs. Formally, as we show in [25], the continuum limit of the coupled system is equivalent to a, possibly infinite, coupled system of PDEs with distributional coefficients. Similarly, a number of authors $[39,46]$ have exploited the equivalence of the reaction-diffusion master equation to a discrete version of the second quantization Fock-space formulation of Doi [14] to study formal representations of the continuum limit of the RDME.

1.1. Background on the Reaction-Diffusion Master Equation. We begin by formulating the reaction-diffusion master equation in Subsection 1.1.1. A recent review of stochastic reaction-diffusion models and numerical methods, including the RDME, is provided in [16]. In Subsection 1.1.2 we present a standard physical argument for determining mesh sizes where the reaction-diffusion master equation should be a "reasonable" physical model. Subsection 1.1.3 briefly reviews the relationship between deterministic reaction-diffusion partial differential equation models and the reaction-diffusion master equation.

1.1.1. Mathematical Formulation. We consider the stochastic reaction and diffusion of chemical species within a domain, $\Omega$. $\Omega$ may denote a closed volume, or all of $\mathbb{R}^{3}$. In the reaction-diffusion master equation model, $\Omega$ is divided by a mesh into a collection voxels labeled by vectors $i$ in some index set $I$ (i.e. $i \in I$ ). For example, if $\Omega=\mathbb{R}^{3}$ then $I=\mathbb{Z}^{3}$. It is assumed that the size of each voxel can be chosen such that within each voxel, independently, the well-mixed formulation of stochastic chemical kinetics [34] is physically valid. Determining for which mesh sizes this supposition is reasonable is one of the main goals of this work, and is further discussed in Sections 1.1.2 and 2. Given this assumption, diffusive transitions of particles between voxels are then modeled as first order chemical reactions. Note that this is equivalent to modeling diffusion as a continuous-time random walk on a lattice.

The state of the chemical system of interest is defined to be the number of each chemical species within each voxel. Let $M_{i}^{l}(t)$ denote the random variable for the 
number of particles of chemical species $l$ in the $\boldsymbol{i}$ 'th voxel, $l=1 \ldots L$. We define $\boldsymbol{M}_{\boldsymbol{i}}(t)=\left(M_{\boldsymbol{i}}^{1}, \ldots, M_{\boldsymbol{i}}^{L}\right)$ to be the state vector of the chemical species in the $\boldsymbol{i}$ 'th voxel, and $\boldsymbol{M}(t)=\left\{\boldsymbol{M}_{\boldsymbol{i}}\right\}_{\boldsymbol{i} \in I}$ to be the total state of the system (i.e. the number of all species at all locations). The probability for $\boldsymbol{M}(t)$ to have the value $\boldsymbol{m}$ at time $t$, given the initial state, $\boldsymbol{M}(0)=\boldsymbol{m}_{0}$, is denoted by

$$
P(\boldsymbol{m}, t) \equiv \operatorname{Prob}\left\{\boldsymbol{M}(t)=\boldsymbol{m} \mid \boldsymbol{M}(0)=\boldsymbol{m}_{0}\right\}
$$

We now define a notation to represent changes of state due to diffusive transitions. Let $1_{\boldsymbol{i}}^{l}$ be the state where the number of all chemical species at all locations is zero, except for the $l^{\prime}$ 'th chemical species at the $\boldsymbol{i}$ 'th location, which is one. (i.e. $\boldsymbol{M}(t)+1_{\boldsymbol{i}}^{l}$ would add one to chemical species $l$ in the voxel labeled by $\boldsymbol{i}) . k_{\boldsymbol{i} \boldsymbol{j}}^{l}$ shall denote the diffusive jump rate for each individual molecule of the $l$ 'th chemical species into voxel $\boldsymbol{i}$ from voxel $\boldsymbol{j}$, for $\boldsymbol{i} \neq \boldsymbol{j}$. Since diffusion is treated as a first order reaction and molecules are assumed to diffuse independently, the total probability per unit time at time $t$ for one molecule of species $l$ to jump from voxel $\boldsymbol{j}$ to voxel $\boldsymbol{i}$ is $k_{\boldsymbol{i j}}^{l} M_{\boldsymbol{j}}^{l}(t) . k_{\boldsymbol{i} \boldsymbol{i}}$ is chosen to be zero, so that a molecule must hop to a different voxel.

We assume there are $K$ possible reactions, with the function $a_{\boldsymbol{i}}^{k}\left(\boldsymbol{m}_{\boldsymbol{i}}\right)$ giving the probability per unit time of reaction $k$ occurring in the $\boldsymbol{i}$ 'th voxel when $\boldsymbol{M}_{\boldsymbol{i}}(t)=\boldsymbol{m}_{\boldsymbol{i}}$. For example, letting $k$ label the unimolecular (first order) reaction $\mathrm{S}^{l} \rightarrow \mathrm{S}^{l^{\prime}}$, then $a_{\boldsymbol{i}}^{k}\left(\boldsymbol{m}_{\boldsymbol{i}}\right)=\alpha m_{\boldsymbol{i}}^{l}$, where $\alpha$ is the rate constant in units of number of occurrences of the reaction per molecule of $\mathrm{S}^{l}$ per unit time. Letting $k^{\prime}$ denote the index of the bimolecular reaction $S^{l}+S^{l^{\prime}} \rightarrow S^{l^{\prime \prime}}$, where $l \neq l^{\prime}$, then $a_{\boldsymbol{i}}^{k^{\prime}}\left(\boldsymbol{m}_{\boldsymbol{i}}\right)=\beta m_{\boldsymbol{i}}^{l} m_{\boldsymbol{i}}^{l^{\prime}}$. Here $\beta$ is the rate constant in units of number of occurrences of the reaction per molecule of $\mathrm{S}^{l}$ and per molecule of $\mathrm{S}^{l^{\prime}}$, per unit time. State changes in $\boldsymbol{M}_{\boldsymbol{i}}(t)$ due to an occurrence of the $k$ 'th chemical reaction in the $\boldsymbol{i}$ 'th mesh voxel will be denoted by the vector $\boldsymbol{\nu}_{k}=\left(\nu_{k}^{1}, \ldots, \nu_{k}^{L}\right)$ (i.e. $\left.\boldsymbol{M}_{\boldsymbol{i}}(t) \rightarrow \boldsymbol{M}_{\boldsymbol{i}}(t)+\boldsymbol{\nu}_{k}\right)$. The corresponding state change in $\boldsymbol{M}(t)$ due to an occurrence of the $k^{\prime}$ th reaction in the $\boldsymbol{i}$ 'th voxel will be denoted by $\boldsymbol{\nu}_{k} 1_{\boldsymbol{i}}$ (i.e. $\left.\boldsymbol{M}(t) \rightarrow \boldsymbol{M}(t)+\boldsymbol{\nu}_{k} 1_{\boldsymbol{i}}\right)$.

With these definitions, the reaction-diffusion master equation for the time evolution of $P(\boldsymbol{m}, t)$ is then

$$
\begin{aligned}
\frac{d P(\boldsymbol{m}, t)}{d t}= & \sum_{\boldsymbol{i} \in I} \sum_{\boldsymbol{j} \in I} \sum_{l=1}^{L}\left(k_{\boldsymbol{i} \boldsymbol{j}}^{l}\left(m_{\boldsymbol{j}}^{l}+1\right) P\left(\boldsymbol{m}+1_{\boldsymbol{j}}^{l}-1_{\boldsymbol{i}}^{l}, t\right)-k_{\boldsymbol{j} \boldsymbol{i}}^{l} m_{\boldsymbol{i}}^{l} P(\boldsymbol{m}, t)\right) \\
& +\sum_{\boldsymbol{i} \in I} \sum_{k=1}^{K}\left(a_{\boldsymbol{i}}^{k}\left(\boldsymbol{m}_{\boldsymbol{i}}-\boldsymbol{\nu}_{k}\right) P\left(\boldsymbol{m}-\boldsymbol{\nu}_{k} 1_{\boldsymbol{i}}, t\right)-a_{\boldsymbol{i}}^{k}\left(\boldsymbol{m}_{\boldsymbol{i}}\right) P(\boldsymbol{m}, t)\right)
\end{aligned}
$$

This is a coupled set of ODEs over all possible non-negative integer values of the matrix $\boldsymbol{m}$. Notice the important point that the reaction probabilities per unit time, $a_{\boldsymbol{i}}^{k}\left(\boldsymbol{m}_{\boldsymbol{i}}\right)$, may depend on spatial location. To the authors' knowledge, this equation goes back to the work of Gardiner [20].

Equation (1.2) is separated into two sums. The first term corresponds to diffusive motion between voxels $\boldsymbol{i}$ and $\boldsymbol{j}$ of a given species, $l$. The second is just the components of the chemical master equation [34], but applied at each individual voxel. In previous work we have shown that, as the mesh spacing approaches zero, to recover diffusion of an individual molecule in a system with no chemical reactions or to recover diffusion of the mean chemical concentration of each species in (1.2), the diffusive jump rates should be chosen so as to determine a discretization of the Laplacian [28]. 
Let $D^{l}$ denote the diffusion constant of chemical species $l$. For a regular Cartesian mesh in $\mathbb{R}^{d}$, the diffusive jump rates for species $l$ would be given by

$$
k_{\boldsymbol{j} \boldsymbol{i}}^{l}= \begin{cases}D^{l} / h^{2}, & \boldsymbol{i} \text { a non-diagonal neighbor of } \boldsymbol{j}, \\ 0, & \text { else. }\end{cases}
$$

Denote by $\boldsymbol{e}_{k}$ the unit vector along the $k^{\prime}$ th coordinate axis of $\mathbb{R}^{d}$. For a Cartesian mesh in $\mathbb{R}^{d}$ the reaction-diffusion master equation (1.2) then simplifies to

$$
\begin{aligned}
\frac{d P(\boldsymbol{m}, t)}{d t}= & \sum_{\boldsymbol{i} \in \mathbb{Z}^{d}} \sum_{k=1}^{d} \sum_{ \pm} \sum_{l=1}^{L} \frac{D^{l}}{h^{2}}\left(\left(m_{\boldsymbol{i} \pm \boldsymbol{e}_{k}}^{l}+1\right) P\left(\boldsymbol{m}+1_{\boldsymbol{i} \pm \boldsymbol{e}_{k}}^{l}-1_{\boldsymbol{i}}^{l}, t\right)-m_{\boldsymbol{i}}^{l} P(\boldsymbol{m}, t)\right) \\
& +\sum_{\boldsymbol{i} \in \mathbb{Z}^{d}} \sum_{k=1}^{K}\left(a_{\boldsymbol{i}}^{k}\left(\boldsymbol{m}_{\boldsymbol{i}}-\boldsymbol{\nu}_{k}\right) P\left(\boldsymbol{m}-\boldsymbol{\nu}_{k} 1_{\boldsymbol{i}}, t\right)-a_{\boldsymbol{i}}^{k}\left(\boldsymbol{m}_{\boldsymbol{i}}\right) P(\boldsymbol{m}, t)\right) .
\end{aligned}
$$

1.1.2. Physical Validity. To date, no rigorous derivation of the reaction-diffusion master equation from a more microscopic physical model has been given. One systematic computational study was reported in [9] showing good agreement between the reaction-diffusion master equation and Boltzmann-like dynamics. The validity of the reaction-diffusion master equation model is often assumed based on the physical argument presented below (see, for example, the supplement to [15]).

First order reactions are assumed to represent internal events, and as such are presupposed to be independent of diffusion. We also assume that on relevant spatial scales of interest, molecular interaction forces are weak, so that until two molecules are sufficiently close they do not influence each other's movement. Motion of molecules is then taken to be purely diffusive. To ensure that the continuous-time random walk approximation to diffusion inherent in the reaction-diffusion master equation is accurate, we must chose the mesh spacing significantly smaller than characteristic length scales of interest. Denoting this length scale by $L$, and the width of a (cubic) voxel by $h$, we then require

$$
L \gg h
$$

The primary physical assumption in formulating the reaction-diffusion master equation is that a separation of timescales exists such that on the spatial scale of voxels bimolecular reactions may be treated as well-mixed. For example, consider the bimolecular reaction $\mathrm{A}+\mathrm{B} \rightarrow \mathrm{C}$ with rate constant $K$. It is assumed that within a given voxel the timescale of a well-mixed bimolecular reaction between one specific molecule of chemical species A and any molecule of species $\mathrm{B}, \tau_{K_{\mathrm{A}}}$, is much larger than the timescale for the A molecule and an arbitrary B molecule to become well-mixed relative to each other due to diffusion, $\tau_{D}$. (Here $D=D^{\mathrm{A}}+D^{\mathrm{B}}$ denotes the relative diffusion constant between the $\mathrm{A}$ and $\mathrm{B}$ molecules). We specifically assume that

$$
\tau_{K_{\mathrm{A}}} \gg \tau_{D},
$$

where

$$
\tau_{K_{\mathrm{A}}} \approx \frac{1}{K[B]}, \quad \tau_{D} \approx \frac{h^{2}}{D}
$$


Letting $n_{\mathrm{B}}$ denote the number of $\mathrm{B}$ molecules inside the voxel, than in three-dimensions $[\mathrm{B}]=n_{\mathrm{B}} / h^{3}$, so that equation (1.5) simplifies to

$$
h \gg \frac{K n_{\mathrm{B}}}{D} .
$$

Combining this with equation (1.4) we have that

$$
L \gg h \gg \frac{K n_{\mathrm{B}}}{D} .
$$

It is therefore necessary to bound $h$ from above and below to ensure accuracy of the reaction-diffusion master equation.

1.1.3. Relation to Deterministic Reaction-Diffusion PDEs. We now examine the relation between the reaction diffusion master equation and standard deterministic reaction-diffusion PDE models. Define $V_{\boldsymbol{i}}$ to be the volume of the $\boldsymbol{i}$ 'th voxel. We let $C_{i}^{l}(t)=M_{i}^{l}(t) / V_{i}$ be the random variable for the chemical concentration of species $l$, in voxel $\boldsymbol{i}$, and define $\boldsymbol{C}_{\boldsymbol{i}}(t)=\left(C_{\boldsymbol{i}}^{1}, \ldots, C_{\boldsymbol{i}}^{L}\right)$. Denote by $\tilde{a}_{\boldsymbol{i}}^{k}$ the concentration dependent form of $a_{\boldsymbol{i}}^{k}$. $\tilde{a}_{\boldsymbol{i}}^{k}$ and $a_{\boldsymbol{i}}^{k}$ are related by $\tilde{a}_{\boldsymbol{i}}^{k}(\boldsymbol{c})=a_{\boldsymbol{i}}^{k}\left(V_{\boldsymbol{i}} \boldsymbol{c}\right) / V_{\boldsymbol{i}}$, and vice-versa $a_{\boldsymbol{i}}^{k}(\boldsymbol{m})=\tilde{a}_{\boldsymbol{i}}^{k}\left(\boldsymbol{m} / V_{\boldsymbol{i}}\right) V_{\boldsymbol{i}}$. Letting $\mathbb{E}\left[C_{\boldsymbol{i}}^{l}(t)\right]$ denote the average value of $C_{\boldsymbol{i}}^{l}(t)$, from equation (1.2) we then find

$$
\frac{d \mathbb{E}\left[C_{\boldsymbol{i}}^{l}\right]}{d t}=\sum_{\boldsymbol{j} \in I}\left(\frac{V_{\boldsymbol{j}}}{V_{\boldsymbol{i}}} k_{\boldsymbol{i} \boldsymbol{j}}^{l} \mathbb{E}\left[C_{\boldsymbol{j}}^{l}\right]-k_{\boldsymbol{j} \boldsymbol{i}}^{l} \mathbb{E}\left[C_{\boldsymbol{i}}^{l}\right]\right)+\sum_{k=1}^{K} \nu_{k}^{l} \mathbb{E}\left[\tilde{a}_{\boldsymbol{i}}^{k}\left(C_{\boldsymbol{i}}\right)\right] .
$$

Note the important point that for nonlinear reactions, such as bimolecular reactions,

$$
\mathbb{E}\left[\tilde{a}_{i}^{k}\left(C_{\boldsymbol{i}}(t)\right)\right] \neq \tilde{a}_{\boldsymbol{i}}^{k}\left(\mathbb{E}\left[C_{\boldsymbol{i}}(t)\right]\right) .
$$

For chemical systems in which any nonlinear reactions are present, the equations for the mean concentrations will then be coupled to an infinite set of ODEs for the higher order moments.

We now consider the continuum limit that $h \rightarrow 0$. Let $\boldsymbol{x}$ denote the centroid of the voxel labeled by $\boldsymbol{i}$, and assume that $h$ is chosen to approach zero such that $\boldsymbol{x}$ always remains the centroid of some voxel. We then define

$$
S^{l}(\boldsymbol{x}, t)=\lim _{h \rightarrow 0} \mathbb{E}\left[C_{\boldsymbol{i}}^{l}(t)\right]
$$

and $\boldsymbol{S}(\boldsymbol{x}, t)=\left(S^{1}(\boldsymbol{x}, t), \ldots, S^{L}(\boldsymbol{x}, t)\right)$. Denote by $D^{l}$ the diffusion constant of the $l$ 'th chemical species, and define $\tilde{a}^{k}(\boldsymbol{S}(\boldsymbol{x}, t), \boldsymbol{x})$ to be the continuum spatially varying concentration dependent form of $a_{i}^{k}$. Following the discussion in Subsection 1.1.1, the jump rates $k_{\boldsymbol{i} \boldsymbol{j}}^{l}$ are chosen to be a discretization of the Laplacian. The deterministic reaction-diffusion $\mathrm{PDE}$ model can be thought of as the approximation that

$$
\frac{\partial S^{l}(\boldsymbol{x}, t)}{\partial t}=D^{l} \Delta S^{l}+\sum_{k=1}^{K} \nu_{k}^{l} \tilde{a}^{k}(\boldsymbol{S}(\boldsymbol{x}, t), \boldsymbol{x}) .
$$

This equation implicitly assumes that in the formal continuum limit the equations for the mean concentrations form a closed system. In general, however, this is only true for chemical systems in which all reaction terms are linear due to equation (1.7). For systems with nonlinear reaction terms, the equations for the mean concentrations 
would then remain coupled to higher order moments in the formal continuum limit, giving an infinite system of equations to solve in order to determine the means.

As discussed in the introduction, it has been shown more generally that the formal continuum limit of the reaction-diffusion master equation itself may be interpreted as a Fock Space representation of a quantum field theory [39].

2. A Reduced Model To Study $h$ Dependence of RDME. We now investigate the behavior of the reaction-diffusion master equation as the mesh spacing, $h$, becomes small in a simplified model. The simplified model studied is that of two molecules, one of chemical species $A$ and one of chemical species $B$, that diffuse in $\mathbb{R}^{3}$ and can annihilate by undergoing the chemical reaction $\mathrm{A}+\mathrm{B} \rightarrow \varnothing$. In this system, the reaction-diffusion master equation can be reduced to a form that is much easier to study analytically than equation (1.3). (Note that we subsequently assume we are working in $\mathbb{R}^{3}$ with a standard cubic Cartesian mesh of mesh width $h$ ). We show that in this special case the continuum limit is formally given by a partial differential equation with distributional coefficients.

The model problem can be derived from the reaction-diffusion master equation (1.3) as follows. We first simplify to the reaction $\mathrm{A}+\mathrm{B} \rightarrow \mathrm{C}$ with well-mixed bimolecular reaction rate $k$, and only one molecule of $\mathrm{A}$, one molecule of $\mathrm{B}$, and no molecules of $\mathrm{C}$ initially. $k$ is assumed to have units of volume/time as is standard for deterministic ODE models. We denote by $\boldsymbol{A}(t)=\left\{A_{\boldsymbol{i}}(t)\right\}_{\boldsymbol{i} \in \mathbb{Z}^{3}}$ the vector stochastic process for the number of chemical species $\mathrm{A}$ at each location at time $t$. (We define $\boldsymbol{B}(t)$ and $\boldsymbol{C}(t)$ similarly). $a_{\boldsymbol{i}}$ will denote a specific number of molecules of chemical species A at location $\boldsymbol{i}$, and

$$
\boldsymbol{a}=\left\{a_{i} \mid \boldsymbol{i} \in \mathbb{Z}^{3}\right\},
$$

a possible value of $\boldsymbol{A}(t)$. (We again define $\boldsymbol{b}$ and $\boldsymbol{c}$ similarly). The notation $\boldsymbol{a}+1_{\boldsymbol{i}}$ will, as before, represent $\boldsymbol{a}$ with one added to $a_{\boldsymbol{i}}$. In terms of $\boldsymbol{a}, \boldsymbol{b}$, and $\boldsymbol{c}$, the reactiondiffusion master equation gives the time evolution of

$$
P(\boldsymbol{a}, \boldsymbol{b}, \boldsymbol{c}, t)=\operatorname{Prob}\{\boldsymbol{A}(t)=\boldsymbol{a}, \boldsymbol{B}(t)=\boldsymbol{b}, \boldsymbol{C}(t)=\boldsymbol{c} \mid \boldsymbol{A}(0), \boldsymbol{B}(0), \boldsymbol{C}(0)\} .
$$

Let $\mathbf{0}$ denote the zero vector. We assume that $\boldsymbol{A}(0)=1_{\boldsymbol{i}_{0}}, \boldsymbol{B}(0)=1_{\boldsymbol{i}_{0}^{\prime}}$, and $\boldsymbol{C}(0)=\mathbf{0}$. At time $t$, the state of the chemical system is then $\boldsymbol{A}(t)=1_{\boldsymbol{i}}, \boldsymbol{B}(t)=1_{\boldsymbol{i}^{\prime}}$, and $\boldsymbol{C}(t)=\mathbf{0}$ prior to the reaction occurring, or $\boldsymbol{A}(t)=\mathbf{0}, \boldsymbol{B}(t)=\mathbf{0}$, and $\boldsymbol{C}(t)=1_{\boldsymbol{i}}$ subsequent to the reaction occurring. (Here $\boldsymbol{i}$ and $\boldsymbol{i}^{\prime}$ label arbitrary molecule positions). Let $\delta_{\boldsymbol{i} \boldsymbol{i}^{\prime}}$ denote the three-dimensional Kronecker delta function, zero if $\boldsymbol{i} \neq \boldsymbol{i}^{\prime}$ and one if $\boldsymbol{i}=\boldsymbol{i}^{\prime}$. For this system, the reaction-diffusion master equation (1.3) simplifies to

$$
\begin{aligned}
\frac{d P}{d t}\left(1_{\boldsymbol{i}}, 1_{\boldsymbol{i}^{\prime}}, \mathbf{0}\right)=\sum_{k=1}^{3} \sum_{ \pm}[ & \frac{D^{\mathrm{A}}}{h^{2}}\left(P\left(1_{\boldsymbol{i}_{ \pm} \boldsymbol{e}_{k}}, 1_{\boldsymbol{i}^{\prime}}, \mathbf{0}, t\right)-P\left(1_{\boldsymbol{i}}, 1_{\boldsymbol{i}^{\prime}}, \mathbf{0}, t\right)\right) \\
& \left.+\frac{D^{\mathrm{B}}}{h^{2}}\left(P\left(1_{\boldsymbol{i}}, 1_{\boldsymbol{i}^{\prime} \pm \boldsymbol{e}_{k}}, \mathbf{0}, t\right)-P\left(1_{\boldsymbol{i}}, 1_{\boldsymbol{i}^{\prime}}, \mathbf{0}, t\right)\right)\right] \\
-\frac{k}{h^{3}} \delta_{\boldsymbol{i} \boldsymbol{i}^{\prime}} & P\left(1_{\boldsymbol{i}}, 1_{\boldsymbol{i}^{\prime}}, \mathbf{0}, t\right),
\end{aligned}
$$

for states where a reaction has not yet occurred, and to

$$
\frac{d P}{d t}\left(\mathbf{0}, \mathbf{0}, 1_{i}\right)=\sum_{k=1}^{3} \sum_{ \pm} \frac{D^{\mathrm{C}}}{h^{2}}\left[P\left(\mathbf{0}, \mathbf{0}, 1_{\boldsymbol{i} \pm \boldsymbol{e}_{k}}, t\right)-P\left(\mathbf{0}, \mathbf{0}, 1_{\boldsymbol{i}}, t\right)\right]+\frac{k}{h^{3}} P\left(1_{\boldsymbol{i}}, 1_{\boldsymbol{i}}, \mathbf{0}, t\right)
$$


for states where the reaction has occurred. Note the important point that the bimolecular reaction rate is given by $k / h^{3}$, since $k$ has units of volume/time.

This simplified reaction-diffusion master equation is completely equivalent to a new representation described by the probability distributions $F^{(0,0,1)}(\boldsymbol{i}, t)$ and $F^{(1,1,0)}\left(\boldsymbol{i}, \boldsymbol{i}^{\prime}, t\right)$. Here superscripts denote the total number of each of species A, B, and $\mathrm{C}$ in the system, and indices give the corresponding locations of these molecules. $F^{(1,1,0)}\left(\boldsymbol{i}, \boldsymbol{i}^{\prime}, t\right)$ denotes the probability that the species A and B particles have not yet reacted, and are located in voxels $\boldsymbol{i}$ and $\boldsymbol{i}^{\prime}$ respectively at time $t . F^{(0,0,1)}(\boldsymbol{i}, t)$ gives the probability that the particles have reacted, and the $\mathrm{C}$ particle they created is located in voxel $\boldsymbol{i}$ at time $t$.

Assuming the A particle starts in voxel $\boldsymbol{i}_{0}$ and the $\mathrm{B}$ particle in voxel $\boldsymbol{i}_{0}^{\prime}$, the equations of evolution of $F^{(0,0,1)}(\boldsymbol{i}, t)$ and $F^{(1,1,0)}\left(\boldsymbol{i}, \boldsymbol{i}^{\prime}, t\right)$ follow immediately from the simplified reaction-diffusion master equation, and are given by

$$
\begin{aligned}
\frac{d F^{(1,1,0)}}{d t}\left(\boldsymbol{i}, \boldsymbol{i}^{\prime}, t\right) & =\left(\left[D^{\mathrm{A}} \Delta_{h}^{\mathrm{A}}+D^{\mathrm{B}} \Delta_{h}^{\mathrm{B}}\right] F^{(1,1,0)}\right)\left(\boldsymbol{i}, \boldsymbol{i}^{\prime}, t\right)-\frac{k}{h^{3}} \delta_{\boldsymbol{i} \boldsymbol{i}^{\prime}} F^{(1,1,0)}(\boldsymbol{i}, \boldsymbol{i}, t), \\
\frac{d F^{(0,0,1)}}{d t}(\boldsymbol{i}, t) & =\left(D^{\mathrm{C}} \Delta_{h}^{\mathrm{C}} F^{(0,0,1)}\right)(\boldsymbol{i}, t)+\frac{k}{h^{3}} F^{(1,1,0)}(\boldsymbol{i}, \boldsymbol{i}, t),
\end{aligned}
$$

with initial conditions $F^{(1,1,0)}\left(\boldsymbol{i}, \boldsymbol{i}^{\prime}, 0\right)=\delta_{\boldsymbol{i} i_{0}} \delta_{\boldsymbol{i}^{\prime} \boldsymbol{i}_{0}^{\prime}}$ and $F^{(0,0,1)}(\boldsymbol{i}, 0)=0$. Here $\Delta_{h}^{\mathrm{A}}$ denotes the standard second order discrete Laplacian acting on the coordinates of the A particle, and $\Delta_{h}^{\mathrm{B}}$ denotes the discrete Laplacian acting on the coordinates of the species B particle. For example,

$$
\left(\Delta_{h}^{\mathrm{B}} F^{(1,1,0)}\right)\left(\boldsymbol{i}, \boldsymbol{i}^{\prime}, t\right)=\sum_{k=1}^{3} \sum_{ \pm} \frac{D^{\mathrm{B}}}{h^{2}}\left(F^{(1,1,0)}\left(\boldsymbol{i}, \boldsymbol{i}^{\prime} \pm \boldsymbol{e}_{k}, t\right)-F^{(1,1,0)}\left(\boldsymbol{i}, \boldsymbol{i}^{\prime}, t\right)\right) .
$$

$\Delta_{h}^{\mathrm{C}}$ is defined similarly. More general multi-particle reaction-diffusion master equations can also be converted to related systems of coupled differential-difference equations. These equations correspond to discrete versions of the spatially-continuous "distribution function" stochastic reaction-diffusion model proposed in [14]. Note that if the number of reacting molecules is unbounded, the number of equations will be infinite. See [25] for a derivation of the corresponding system of equations governing the reaction $\mathrm{A}+\mathrm{B} \leftrightarrows \mathrm{C}$ with arbitrary amounts of each chemical species.

Notice that equation (2.1) is independent of equation (2.2), and by itself can be thought of as representing the reaction $\mathrm{A}+\mathrm{B} \rightarrow \varnothing$. To study this chemical reaction we drop the $\mathrm{C}$ dependence in $F^{(1,1,0)}\left(\boldsymbol{i}, \boldsymbol{i}^{\prime}, t\right)$ and study $F^{(1,1)}\left(\boldsymbol{i}, \boldsymbol{i}^{\prime}, t\right)$, which satisfies

$$
\frac{d F^{(1,1)}}{d t}\left(\boldsymbol{i}, \boldsymbol{i}^{\prime}, t\right)=\left(\left[D^{\mathrm{A}} \Delta_{h}^{\mathrm{A}}+D^{\mathrm{B}} \Delta_{h}^{\mathrm{B}}\right] F^{(1,1)}\right)\left(\boldsymbol{i}, \boldsymbol{i}^{\prime}, t\right)-\frac{k}{h^{3}} \delta_{\boldsymbol{i} \boldsymbol{i}^{\prime}} F^{(1,1)}(\boldsymbol{i}, \boldsymbol{i}, t),
$$

with initial condition $F^{(1,1)}\left(\boldsymbol{i}, \boldsymbol{i}^{\prime}, 0\right)=\delta_{\boldsymbol{i} \boldsymbol{i}_{0}} \delta_{\boldsymbol{i}^{\prime} \boldsymbol{i}_{0}^{\prime}}$.

We now consider the separation vector, $\boldsymbol{i}-\boldsymbol{i}^{\prime}$, for the two particles of species A and B. Define the probability of the separation vector having the value $\boldsymbol{j}$,

$$
\begin{aligned}
P(\boldsymbol{j}, t) & =\sum_{\boldsymbol{i}-\boldsymbol{i}^{\prime}=\boldsymbol{j}} F^{(1,1)}\left(\boldsymbol{i}, \boldsymbol{i}^{\prime}, t\right), \\
& =\sum_{\boldsymbol{i} \in Z^{3}} F^{(1,1)}(\boldsymbol{i}, \boldsymbol{i}-\boldsymbol{j}, t) .
\end{aligned}
$$


It follows from (2.3), as shown in [26], that $P(\boldsymbol{j}, t)$ satisfies

$$
\begin{aligned}
\frac{d P}{d t}(\boldsymbol{j}, t) & =\left(D \Delta_{h} P\right)(\boldsymbol{j}, t)-\frac{k}{h^{3}} \delta_{\boldsymbol{j} \mathbf{0}} P(\mathbf{0}, t), \\
P(\boldsymbol{j}, 0) & =\delta_{\boldsymbol{j}_{0}},
\end{aligned}
$$

where $D=D^{\mathrm{A}}+D^{\mathrm{B}}$ and $\boldsymbol{j}_{0}=\boldsymbol{i}_{0}-\boldsymbol{i}_{0}^{\prime}$. Note this equation is equivalent to a reactiondiffusion master equation model of the binding of a single diffusing particle to a fixed binding site at the origin.

To study the limiting behavior of our system for small $h$ we convert equation (2.5) from units of probability to units of probability density. This change is necessary since the underlying SCDLR model we compare with is described by the evolution of a probability density. Let $\boldsymbol{x}_{\boldsymbol{j}}=h \boldsymbol{j}$ denote the center of the Cartesian voxel labeled by $\boldsymbol{j} \in \mathbb{Z}^{3}$. We denote the probability density for the separation vector to be $\boldsymbol{x}_{\boldsymbol{j}}$ at time $t$ by $p_{h}\left(\boldsymbol{x}_{\boldsymbol{j}}, t\right) \equiv P(\boldsymbol{j}, t) / h^{3}$. Equation (2.5) can now be converted to an equation for $p_{h}\left(\boldsymbol{x}_{\boldsymbol{j}}, t\right)$, giving

$$
\begin{aligned}
\frac{d p_{h}}{d t}\left(\boldsymbol{x}_{\boldsymbol{j}}, t\right) & =D\left(\Delta_{h} p_{h}\right)\left(\boldsymbol{x}_{\boldsymbol{j}}, t\right)-\frac{k}{h^{3}} \delta_{j \mathbf{0}} p_{h}(\mathbf{0}, t), \\
p_{h}\left(\boldsymbol{x}_{\boldsymbol{j}}, t\right) & =\frac{1}{h^{3}} \delta_{\boldsymbol{j} j_{0}}, \quad \boldsymbol{j}_{0} \neq \mathbf{0},
\end{aligned}
$$

where again, $\boldsymbol{j}_{0}=\boldsymbol{i}_{0}-\boldsymbol{i}_{0}^{\prime}$. Note the assumption, which we use for the remainder of this paper, that initially the molecules are in different voxels, i.e. $\boldsymbol{j}_{0} \neq \mathbf{0}$. This assumption is necessary to avoid a product of delta functions centered at the same location in the SCDLR model used in Section 2.2. Equation (2.6) is the final reduced form of the reaction $\mathrm{A}+\mathrm{B} \rightarrow \varnothing$ that we subsequently study.

In Section 2.1 we consider the limit of this model as $h \rightarrow 0$ and observe that the molecules never react. In contrast, we show in Section 2.2 that this simplified model can be thought of as a good asymptotic approximation to a specific microscopic continuous-space reaction-diffusion model, assuming $h$ is neither too small nor too large. Specifically, we show that the simplified discrete model can be thought of as an asymptotic approximation to a SCDLR model, where reactions are modeled as occurring instantly when two diffusing particles approach within a specified reaction radius. The asymptotic approximation of (2.6) to the SCDLR model diverges like $1 / h$ as $h \rightarrow 0$ and therefore the master equation loses accuracy when $h$ is sufficiently small. Recall, however, that $h$ cannot be taken arbitrarily large as then neither diffusion nor the reaction process are approximated accurately! In Section 2.2.3 we investigate the error between the asymptotic approximations, truncated after the second order terms, of the SCDLR model and the simplified reaction-diffusion master equation model. Both numerically calculated errors values and analytical convergence/divergence rates are presented. It is shown that for this simplified model, the physically derived bounds on $h$ given in Section 1.1.2 may be reasonable restrictions on how $h$ should be chosen so that the truncated asymptotic expansion of the reaction-diffusion master equation provides an accurate approximation to the truncated expansion of the SCDLR model.

2.1. Continuum Limit as $h \rightarrow 0$. We might expect the solution to equation (2.6) to approach the solution to

$$
\begin{aligned}
\frac{\partial p}{\partial t}(\boldsymbol{x}, t) & =D \Delta p(\boldsymbol{x}, t)-k \delta(\boldsymbol{x}) p(\mathbf{0}, t), \quad \boldsymbol{x} \in \mathbb{R}^{3}, \\
p(\boldsymbol{x}, 0) & =\delta\left(\boldsymbol{x}-\boldsymbol{x}_{0}\right), \quad \boldsymbol{x}_{0} \neq \mathbf{0},
\end{aligned}
$$


as $h \rightarrow 0$. Ignoring, for now, the question of how to define a partial differential equation with distributional coefficients, we next show that as $h \rightarrow 0$ the molecules never react, and simply diffuse relative to each other. Thus, in the continuum limit, the molecules do not feel the delta function reaction term at all.

To study the solution to equation (2.6) as $h \rightarrow 0$ we will make use of the free space Green's function for the discrete-space continuous-time diffusion equation, $G_{h}\left(\boldsymbol{x}_{\boldsymbol{j}}, t\right)$. $G_{h}$ satisfies

$$
\begin{aligned}
\frac{d G_{h}}{d t}\left(\boldsymbol{x}_{\boldsymbol{j}}, t\right) & =D\left(\Delta_{h} G_{h}\right)\left(\boldsymbol{x}_{\boldsymbol{j}}, t\right), \\
G_{h}\left(\boldsymbol{x}_{\boldsymbol{j}}, 0\right) & =\frac{1}{h^{3}} \delta_{\boldsymbol{j} \mathbf{0}},
\end{aligned}
$$

and has the Fourier representation

$$
G_{h}\left(\boldsymbol{x}_{\boldsymbol{j}}, t\right)=\iiint_{\left[\frac{-1}{2 h}, \frac{1}{2 h}\right]^{3}} e^{-4 D t \sum_{k=1}^{3} \sin ^{2}\left(\pi h \xi_{k}\right) / h^{2}} e^{2 \pi i \boldsymbol{\xi} \cdot\left(\boldsymbol{x}_{\boldsymbol{j}}\right)} d \boldsymbol{\xi} .
$$

Here $\boldsymbol{\xi}=\left(\xi_{1}, \xi_{2}, \xi_{3}\right)$, and $[-1 / 2 h, 1 / 2 h]^{3}$ denotes the cube centered at the origin with sides of length $1 / h$. We will also need the Green's function for the continuum free space diffusion equation, $G(\boldsymbol{x}, t)$, given by

$$
G(\boldsymbol{x}, t)=\frac{1}{(4 \pi D t)^{3 / 2}} e^{-|\boldsymbol{x}|^{2} /(4 D t)} .
$$

Note we prove in Theorem A.1 that away from the origin, $G_{h}$ converges to $G$ uniformly in time as $h \rightarrow 0$.

Using Duhammel's Principle, the solution to equation (2.6) may be written as

$$
p_{h}\left(\boldsymbol{x}_{\boldsymbol{j}}, t\right)=G_{h}\left(\boldsymbol{x}_{\boldsymbol{j}}-\boldsymbol{x}_{\boldsymbol{j}_{0}}, t\right)-k \int_{0}^{t} G_{h}\left(\boldsymbol{x}_{\boldsymbol{j}}, t-s\right) p_{h}(\mathbf{0}, s) d s .
$$

Letting $\boldsymbol{x}_{\boldsymbol{j}}=0$, we find that the solution at the origin satisfies the Volterra integral equation of the second kind

$$
p_{h}(\mathbf{0}, t)=G_{h}\left(\boldsymbol{x}_{\boldsymbol{j}_{0}}, t\right)-k \int_{0}^{t} G_{h}(\mathbf{0}, t-s) p_{h}(\mathbf{0}, s) d s,
$$

where we have used that the $G_{h}\left(\boldsymbol{x}_{\boldsymbol{j}}-\boldsymbol{x}_{\boldsymbol{j}_{0}}, t\right)=G_{h}\left(\boldsymbol{x}_{\boldsymbol{j}_{0}}-\boldsymbol{x}_{\boldsymbol{j}}, t\right)$. Note that since $p_{h}(\mathbf{0}, t)=P(\mathbf{0}, t) / h^{3}$, and $P(\boldsymbol{x}, t)$ is the probability distribution for a discrete space continuous-time Markov process, we have that $p_{h}(\mathbf{0}, t) \geq 0$.

We will also find it useful to consider the binding time distribution for the particles, $F_{h}(t)$. Denote by $T$ the random variable for the binding time of the particles; then $F_{h}(t)=\operatorname{Prob}\{T<t\}$ and is given by

$$
\begin{aligned}
F_{h}(t) & \equiv k \int_{0}^{t} p_{h}(\mathbf{0}, s) d s \\
& =\frac{k}{h^{3}} \int_{0}^{t} P(\mathbf{0}, s) d s .
\end{aligned}
$$

Note $F_{h}(t)$ may be defective, i.e. $F_{h}(\infty)<1$, since in three dimensions the particle separation is not guaranteed to ever take the value $\mathbf{0}$ as $t \rightarrow \infty$. Considering the coupled system for both $p_{h}$ and $F_{h}$, total probability is now conserved so that

$$
\sum_{\boldsymbol{j} \in \mathbb{Z}^{3}}\left(p_{h}\left(\boldsymbol{x}_{\boldsymbol{j}}, t\right) h^{3}\right)+F_{h}(t)=1, \quad \forall t \geq 0 .
$$


For the remainder of this section we assume that $\boldsymbol{x}=\boldsymbol{x}_{\boldsymbol{j}}=h \boldsymbol{j}$ for some $\boldsymbol{j} \in \mathbb{Z}^{3}$, and remains fixed as $h \rightarrow 0$. (Likewise we assume that $\boldsymbol{x}_{0}=\boldsymbol{x}_{\boldsymbol{j}_{0}}=h \boldsymbol{j}_{0}$, for some $j_{0} \in \mathbb{Z}^{3}$ and is also held fixed as $h \rightarrow 0$ ). With the preceding definitions, we now show that reaction effects are lost as $h \rightarrow 0$.

THEOREM 2.1. Assume the initial particle separation, $\boldsymbol{x}_{0} \neq \mathbf{0}$, and is held fixed as $h \rightarrow 0$. For all $t \geq 0$, the probability the particles have reacted by time $t$ approaches zero as $h \rightarrow 0$, i.e.

$$
\lim _{h \rightarrow 0} F_{h}(t)=0 .
$$

In addition, assume $\boldsymbol{x} \neq \mathbf{0}$, and is held fixed as $h \rightarrow 0$. Then for all $t>0$, the solution to equation (2.11) converges to the solution to the free space diffusion equation, i.e.

$$
\lim _{h \rightarrow 0} p_{h}(\boldsymbol{x}, t)=G\left(\boldsymbol{x}-\boldsymbol{x}_{0}, t\right), \quad \forall t>0 .
$$

Theorem 2.1 implies that, in the continuum limit, the particles never react and simply diffuse relative to each other. Figure 2.1 shows solution curves as $h$ is varied of $p_{h}(\mathbf{0}, t)$ in Figure 2.1a, and $p_{h}(\boldsymbol{x}, t)$ in Figure 2.1b. A stronger result than the theorem is illustrated in Figure 2.2, where the numerical convergence of $p_{h}(\mathbf{0}, t)$ to zero and $p_{h}(\boldsymbol{x}, t)$ to $G\left(\boldsymbol{x}-\boldsymbol{x}_{0}, t\right)$ are illustrated as functions of $h$. We were unable to calculate $p_{h}(\mathbf{0}, t)$ for sufficiently small mesh widths, $h$, to resolve the asymptotic convergence rate of $p_{h}(\mathbf{0}, t)$ to zero, but the figure shows the decrease in $p_{h}(\mathbf{0}, t)$ as $h$ is decreased. An apparent second order convergence rate of $p_{h}(\boldsymbol{x}, t)$ to $G\left(\boldsymbol{x}-\boldsymbol{x}_{0}, t\right)$ is also seen, though this convergence rate may not be the correct asymptotic rate (since to calculate $p_{h}(\boldsymbol{x}, t)$ we make use of $p_{h}(\mathbf{0}, t)$ through equation (2.11)). Details of the numerical methods used to find $p_{h}(\mathbf{0}, t)$ and $p_{h}(\boldsymbol{x}, t)$ may be found in Appendix B.

To prove Theorem 2.1 we need the following two lemmas and Theorem A.1, which proves that away from the origin $G_{h}$ converges to $G$ uniformly in $t$ as $h \rightarrow 0$.

Lemma 2.2. Assume $\boldsymbol{x} \neq \mathbf{0}$, and $\boldsymbol{x}$ is fixed as $h$ varies. Then for all $\epsilon>0$ there exists an $h_{0}>0$ such that for all $h \leq h_{0}$,

$$
G_{h}(\boldsymbol{x}, t) \leq G(\boldsymbol{x}, t)+\epsilon .
$$

Moreover, for $h \leq h_{0}$

$$
\sup _{t \geq 0} G_{h}(\boldsymbol{x}, t) \leq C
$$

where $C$ is a constant depending only on $\boldsymbol{x}$ (independent of $h$ and $t$ ).

Proof. In Theorem A.1 we prove that $G_{h}(\boldsymbol{x}, t) \rightarrow G(\boldsymbol{x}, t)$ uniformly in $t$. Hence for all $\epsilon>0$, we can find an $h_{0}>0$ such that for all $h \leq h_{0}$,

$$
G_{h}(\boldsymbol{x}, t) \leq G(\boldsymbol{x}, t)+\epsilon .
$$

$G(\boldsymbol{x}, t)$ is maximized for $t=|\boldsymbol{x}|^{2} / 6 D$, so that

$$
\sup _{t \geq 0} G_{h}(\boldsymbol{x}, t) \leq\left(\frac{3}{2 \pi}\right)^{\frac{3}{2}} \frac{1}{|\boldsymbol{x}|^{3}} e^{-3 / 2}+\epsilon .
$$




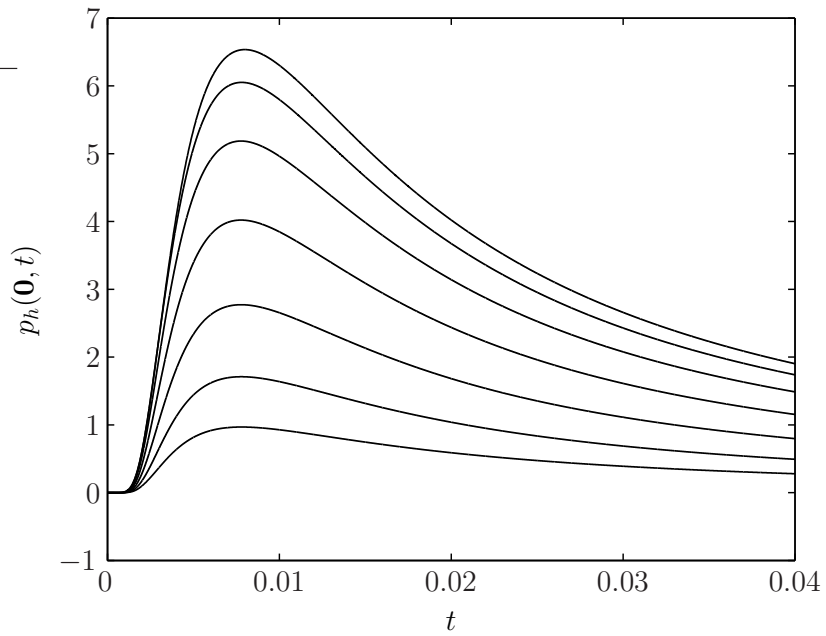

(a)

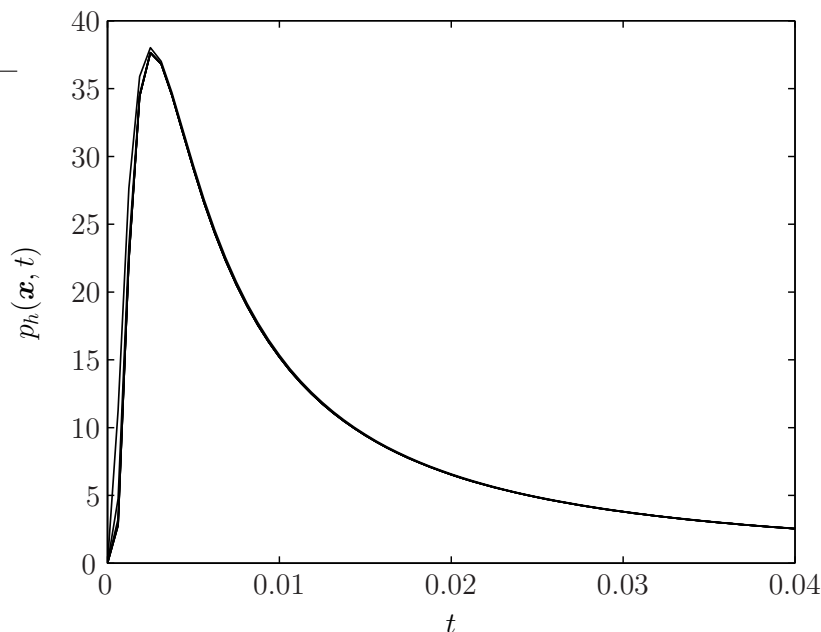

(b)

Fig. 2.1: 2.1a shows $p_{h}(0, t)$ vs. $t$ on $[0, .04]$. Each curve on the figure corresponds to $p_{h}(0, t)$ for a different value of $h$. The topmost curve corresponds to $h=2^{-5}$, the next largest to $2^{-6}$, and so on through the bottom curve, corresponding to $h=2^{-11}$. Figure $2.1 \mathrm{~b}$ shows $p_{h}(\boldsymbol{x}, t)$ vs $t$ on $[0, .04]$ at $\boldsymbol{x}=(0,1 / 8,1 / 8)$. Again curves are plotted for $h=2^{-5}, 2^{-6}, \ldots, 2^{-11}$, however they are visually indistinguishable. In both figures $\boldsymbol{x}_{0}=(1 / 8,1 / 8,1 / 8), D=1$, and $k=4 \pi D a$, where $a=.001$.

We will subsequently make use of the Laplace transform, defined for a function $f(t)$ as

$$
\tilde{f}(s)=\int_{0}^{\infty} f(t) e^{-s t} d t
$$

The second lemma we need is 


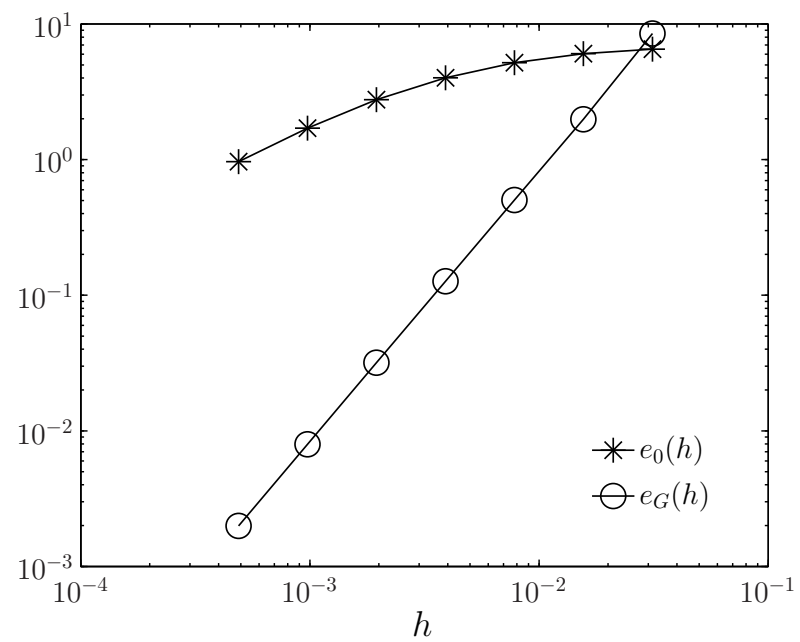

Fig. 2.2: Convergence of $p_{h}(\mathbf{0}, t)$ to zero and $p_{h}(\boldsymbol{x}, t)$ to $G\left(\boldsymbol{x}-\boldsymbol{x}_{0}, t\right)$ as $h \rightarrow 0$. $e_{0}(h)=\max _{t \in[0, .04]} p_{h}(\mathbf{0}, t)$, and $e_{G}(h)=\max _{t \in[0, .04]}\left|p_{h}(\boldsymbol{x}, t)-G\left(\boldsymbol{x}-\boldsymbol{x}_{0}, t\right)\right|$. Note that the slope of the best fit line to $e_{G}(h)=2.0035$. Values of $\boldsymbol{x}, \boldsymbol{x}_{0}, D$, and $k$ are the same as in Figure 2.1.

LEMma 2.3. Denote by $\tilde{G}_{h}(\boldsymbol{x}, s)$ the Laplace transform of $G_{h}(\boldsymbol{x}, t)$ with respect to $t$. We again assume that $\boldsymbol{x} \neq \mathbf{0}$, and $\boldsymbol{x}$ is fixed as $h \rightarrow 0$. Then

$$
\lim _{h \rightarrow 0} \tilde{G}_{h}(\boldsymbol{x}, s)=\tilde{G}(\boldsymbol{x}, s), \quad \forall s>0
$$

and

$$
\lim _{h \rightarrow 0} \tilde{G}_{h}(\mathbf{0}, s)=\infty, \quad \forall s>0 .
$$

Proof. By Theorem A.1, for each fixed $s>0, G_{h}(\boldsymbol{x}, t) e^{-s t}$ converges uniformly in $t$ to $G(\boldsymbol{x}, t) e^{-s t}$ as $h \rightarrow 0$. We may thus conclude that

$$
\lim _{h \rightarrow 0} \int_{0}^{\infty} G_{h}(\boldsymbol{x}, t) e^{-s t} d t=\int_{0}^{\infty} G(\boldsymbol{x}, t) e^{-s t} d t
$$

By definition, this implies that $\tilde{G}_{h}(\boldsymbol{x}, s) \rightarrow \tilde{G}(\boldsymbol{x}, s)$ for all $s>0$, as $h \rightarrow 0$.

For the second limit, we have that for all $t>0, G_{h}(\mathbf{0}, t) \rightarrow G(0, t)=1 /(4 \pi D t)^{3 / 2}$ as $h \rightarrow 0$ by Theorem A.1. Therefore, by Fatou's Lemma,

$$
\begin{aligned}
\liminf _{h \rightarrow 0} \int_{0}^{\infty} G_{h}(\mathbf{0}, t) e^{-s t} d t & \geq \int_{0}^{\infty} \liminf _{h \rightarrow 0} G_{h}(\mathbf{0}, t) e^{-s t} d t \\
& =\int_{0}^{\infty} \frac{1}{(4 \pi D t)^{3 / 2}} e^{-s t} d t \\
& =\infty
\end{aligned}
$$

$\square$ 
With these lemmas, we may now prove the main theorem of this section.

Proof. [Proof of Theorem 2.1] Taking the Laplace Transform of equation (2.12) we find

$$
\tilde{p}_{h}(\mathbf{0}, s)=\frac{\tilde{G}_{h}\left(\boldsymbol{x}_{0}, s\right)}{1+k \tilde{G}_{h}(\mathbf{0}, s)} .
$$

Lemma 2.3 then implies

$$
\lim _{h \rightarrow 0} \tilde{p}_{h}(\mathbf{0}, s)=0, \quad \forall s>0 .
$$

By $(2.13), k p_{h}(\mathbf{0}, t)$ is the binding time density corresponding to the binding time distribution, $F_{h}(t)$. Since $k \tilde{p}_{h}(\mathbf{0}, s) \rightarrow 0$ as $h \rightarrow 0$, the continuity theorem [18, Theorem 2a Section XIII.2] implies that

$$
\lim _{h \rightarrow 0} F_{h}(t)=0 .
$$

Equation (2.11) implies

$$
\left|p_{h}(\boldsymbol{x}, t)-G_{h}\left(\boldsymbol{x}-\boldsymbol{x}_{0}, t\right)\right| \leq k \int_{0}^{t} G_{h}(\boldsymbol{x}, t-s) p_{h}(\mathbf{0}, s) d s .
$$

For all $h$ sufficiently small, Lemma 2.2 implies

$$
\begin{aligned}
\left|p_{h}(\boldsymbol{x}, t)-G_{h}\left(\boldsymbol{x}-\boldsymbol{x}_{0}, t\right)\right| & \leq k\left(\sup _{t} G(\boldsymbol{x}, t)+\epsilon\right) \int_{0}^{t} p_{h}(\mathbf{0}, s) d s \\
& =\left(\sup _{t} G(\boldsymbol{x}, t)+\epsilon\right) F_{h}(t)
\end{aligned}
$$

$F_{h}(t)$ goes to zero and $G_{h}\left(\boldsymbol{x}-\boldsymbol{x}_{0}, t\right) \rightarrow G\left(\boldsymbol{x}-\boldsymbol{x}_{0}, t\right)$ as $h \rightarrow 0$ by Theorem A.1, so that we may conclude $p_{h}(\boldsymbol{x}, t) \rightarrow G\left(\boldsymbol{x}-\boldsymbol{x}_{0}, t\right)$ as $h \rightarrow 0$.

2.2. RDME as an Asymptotic Approximation of Diffusion to a Small Target. While reaction effects are lost as $h \rightarrow 0$, we will now show that for $h$ small, but not "too" small, the simplified model given by equation (2.5) provides an approximation to a SCDLR model. We consider a system consisting of two diffusing molecules, one of species $\mathrm{A}$ and one of species $\mathrm{B}$. The reaction $\mathrm{A}+\mathrm{B} \rightarrow \varnothing$ is modeled by having the two molecules annihilate instantly when they reach a certain physical separation length, called the reaction-radius, and denoted by $a$. We define $f^{(1,1)}\left(\boldsymbol{q}^{\mathrm{A}}, \boldsymbol{q}^{\mathrm{B}}, t\right)$ to represent the probability density for both molecules to exist, the A molecule to be at $\boldsymbol{q}^{\mathrm{A}}$, and the B molecule to be at $\boldsymbol{q}^{\mathrm{B}}$ at time $t$. The model is then

$$
\begin{array}{rlrl}
\frac{\partial f^{(1,1)}}{\partial t}\left(\boldsymbol{q}^{\mathrm{A}}, \boldsymbol{q}^{\mathrm{B}}, t\right) & =\left(\left[D^{\mathrm{A}} \Delta^{\mathrm{A}}+D^{\mathrm{B}} \Delta^{\mathrm{B}}\right] f^{(1,1)}\right)\left(\boldsymbol{q}^{\mathrm{A}}, \boldsymbol{q}^{\mathrm{B}}, t\right), & & \left|\boldsymbol{q}^{\mathrm{A}}-\boldsymbol{q}^{\mathrm{B}}\right|>a, \\
f^{(1,1)}\left(\boldsymbol{q}^{\mathrm{A}}, \boldsymbol{q}^{\mathrm{B}}, t\right) & =0, & & \left|\boldsymbol{q}^{\mathrm{A}}-\boldsymbol{q}^{\mathrm{B}}\right|=a, \\
\lim _{\left|\boldsymbol{q}^{\mathrm{A}}\right| \rightarrow \infty} f^{(1,1)}\left(\boldsymbol{q}^{\mathrm{A}}, \boldsymbol{q}^{\mathrm{B}}, t\right) & =0, & \\
\lim _{\left|\boldsymbol{q}^{\mathrm{B}}\right| \rightarrow \infty} f^{(1,1)}\left(\boldsymbol{q}^{\mathrm{A}}, \boldsymbol{q}^{\mathrm{B}}, t\right) & =0, & & \boldsymbol{q}_{0}^{\mathrm{A}} \neq \boldsymbol{q}_{0}^{\mathrm{B}} .
\end{array}
$$


For simplicity, we again convert to the system for the separation vector, $\boldsymbol{x}=\boldsymbol{q}^{\mathrm{A}}-\boldsymbol{q}^{\mathrm{B}}$, between the A and B particles. Let $p(\boldsymbol{x}, t)$ represent the probability density that the particles have the separation vector $\boldsymbol{x}$ at time $t . p(\boldsymbol{x}, t)$ then satisfies

$$
\begin{aligned}
\frac{\partial p}{\partial t}(\boldsymbol{x}, t) & =D \Delta p(\boldsymbol{x}, t), & & |\boldsymbol{x}|>a, \\
p(\boldsymbol{x}, t) & =0, & & |\boldsymbol{x}|=a, \\
\lim _{|\boldsymbol{x}| \rightarrow \infty} p(\boldsymbol{x}, t) & =0, & & \\
p(\boldsymbol{x}, 0) & =\delta\left(\boldsymbol{x}-\boldsymbol{x}_{0}\right), & & \boldsymbol{x}_{0} \neq \mathbf{0},
\end{aligned}
$$

where $D=D^{\mathrm{A}}+D^{\mathrm{B}}$, and $\boldsymbol{x}_{0}=\boldsymbol{q}_{0}^{\mathrm{A}}-\boldsymbol{q}_{0}^{\mathrm{B}}$. We subsequently refer to equation (2.19) as the SCDLR model.

Recall the definition of $p_{h}\left(\boldsymbol{x}_{\boldsymbol{j}}, t\right)$, the probability density for the particle separation from the master equation to be $\boldsymbol{x}_{\boldsymbol{j}}$ at time $t$, equation (2.6). (Where $\boldsymbol{x}_{\boldsymbol{j}}=h \boldsymbol{j}, \boldsymbol{j} \in \mathbb{Z}^{3}$ ). We expect that $p_{h}\left(\boldsymbol{x}_{\boldsymbol{j}}, t\right) \approx p\left(\boldsymbol{x}_{\boldsymbol{j}}, t\right)$ for $h$ small, but not "too small".

Our main assumption is that $h \gg a$, motivated by the simplification of the heuristic physical assumption, equation (1.6), in the case of one particle of chemical species A and one particle of chemical species B,

$$
h \gg \frac{k}{D} .
$$

We relate the reaction-radius, $a$, to $k / D$ through the definition

$$
a=\frac{k}{4 \pi D} \text {. }
$$

This definition agrees with the well-known form of the bimolecular reaction rate constant for a strongly diffusion limited reaction (see for example [29] for a review of the relevant theory and [43] for the original work). Our key assumption is that $k / D$ is a small parameter, relative to spatial scales of interest, that determines the size of the reaction radius in equation $(2.19)$.

Replacing $k$ with $4 \pi D a$, equation (2.6) becomes

$$
\begin{aligned}
\frac{d p_{h}}{d t}\left(\boldsymbol{x}_{\boldsymbol{j}}, t\right) & =D\left(\Delta_{h} p_{h}\right)\left(\boldsymbol{x}_{\boldsymbol{j}}, t\right)-\frac{4 \pi D a}{h^{3}} \delta_{\boldsymbol{j} \mathbf{0}} p_{h}(\mathbf{0}, t), \\
p_{h}\left(\boldsymbol{x}_{\boldsymbol{j}}, 0\right) & =\frac{1}{h^{3}} \delta_{\boldsymbol{j} \boldsymbol{j}_{0}},
\end{aligned}
$$

where again, $\boldsymbol{j}_{0}=\boldsymbol{i}_{0}-\boldsymbol{i}_{0}^{\prime}$. It is this equation we compare to the SCDLR model, equation (2.19).

As we showed in Section 2.1, the solutions to (2.20) converge to the solutions of the free-space diffusion equation as $h \rightarrow 0$. To investigate the regime where $h$ is small, but $h \gg a$, we introduce asymptotic expansions of equations (2.19) and (2.20) for $a$ small. Our motivation in comparing the asymptotic expansions of the exact solution to (2.19) and the reaction-diffusion master equation (2.20) is in part due to the asymptotic nature of the solution to the formal continuum limit of (2.20). As mentioned in Subsection 2.1, we might expect the solution of the discrete model to approach the solution to

$$
\begin{aligned}
\frac{\partial p}{\partial t}(\boldsymbol{x}, t) & =D \Delta p(\boldsymbol{x}, t)-4 \pi D a \delta(\boldsymbol{x}) p(\mathbf{0}, t), \quad \boldsymbol{x} \in \mathbb{R}^{3}, \\
p(\boldsymbol{x}, 0) & =\delta\left(\boldsymbol{x}-\boldsymbol{x}_{0}\right), \quad \boldsymbol{x}_{0} \neq \mathbf{0},
\end{aligned}
$$


as $h \rightarrow 0$. It is true in the distributional sense that the reaction operator

$$
-4 \pi D a \frac{\delta_{\boldsymbol{j} 0}}{h^{3}} \rightarrow-4 \pi D a \delta(\boldsymbol{x}),
$$

as $h \rightarrow 0$, however, as we saw in Section 2.1 in the continuum limit all reaction effects are lost from the discrete equation (2.20). As described in the introduction, the reaction term in equation (2.21) may be rigorously treated by defining the entire operator in (2.21) as a member of a one-parameter family of self-adjoint extensions to $\mathbb{R}^{3}$ of the Laplacian on $\mathbb{R}^{3} \backslash \mathbf{0}$, see $[3,4,5]$. We denote this family of extensions by the operator $\Delta+\alpha \delta(\boldsymbol{x})$, where $\alpha$ denotes the arbitrary parameter. The solution to (2.21) with the rigorously defined operator $D \Delta-4 \pi D a \delta(\boldsymbol{x})$ [ 5 , Introduction] is the same as the solution to the following pseudo-potential model [19, 24]

$$
\begin{aligned}
\frac{\partial \rho}{\partial t}(\boldsymbol{x}, t) & =D \Delta \rho(\boldsymbol{x}, t)-4 \pi D a \delta(\boldsymbol{x}) \frac{\partial}{\partial r}(r \rho(\boldsymbol{x}, t)), \quad \boldsymbol{x} \in \mathbb{R}^{3}, \\
\rho(\boldsymbol{x}, 0) & =\delta\left(\boldsymbol{x}-\boldsymbol{x}_{0}\right), \quad \boldsymbol{x}_{0} \neq \mathbf{0},
\end{aligned}
$$

where $r=|\boldsymbol{x}|$. These delta function and pseudo-potential operators were introduced in quantum mechanics to give local potentials whose scattering approximates that of a hard sphere of radius $a$. The solution to (2.22) is an asymptotic approximation in $a$ of the solution to the SCDLR model (2.19), accurate through terms of order $a^{2}$ (see for example [27] and compare with the results of Subsection 2.2.2). This suggests that the reaction-diffusion master equation (2.20) provides an approximation to (2.21) and (2.22), and therefore to the SCDLR model (2.19), even though, as shown in Subsection 2.1, it converges to the diffusion equation (i.e. the $\alpha=0$ case), as the mesh spacing approaches zero.

In Section 2.2.1 we derive, through second order, the asymptotic expansion of the discrete reaction diffusion master equation model (2.20), while in Section 2.2.2 we calculate the corresponding expansion of the SCDLR model (2.19). The error between terms of the same order in each of the two expansions is examined in Section 2.2.3. In addition, we also examine the relative error between the expansions, truncated after the second order terms, of the solutions to (2.19) and (2.20).

2.2.1. Perturbation Theory for RDME. In order to examine the intermediate situation that $h$ is small, but $h \gg a$, we now look at the asymptotics of the solution to (2.20) for $a$ small. We begin by calculating the perturbation expansion of $p_{h}(\boldsymbol{x}, t)$ for $a$ small. Throughout this section we assume that $\boldsymbol{x}=\boldsymbol{x}_{\boldsymbol{j}}=h \boldsymbol{j}$ for some $\boldsymbol{j} \in \mathbb{Z}^{3}$, and $\boldsymbol{x}_{0}=\boldsymbol{x}_{\boldsymbol{j}_{0}}=h \boldsymbol{j}_{0}$, for some $\boldsymbol{j}_{0} \in \mathbb{Z}^{3}$. We also assume that $\boldsymbol{x} \neq \mathbf{0}$ and $\boldsymbol{x}_{0} \neq \mathbf{0}$. Using Duhammel's Principle, the solution to equation (2.20) satisfies

$$
p_{h}(\boldsymbol{x}, t)=G_{h}\left(\boldsymbol{x}-\boldsymbol{x}_{0}, t\right)-4 \pi D a \int_{0}^{t} G_{h}(\boldsymbol{x}, t-s) p_{h}(\mathbf{0}, s) d s .
$$

We find an asymptotic expansion of $p_{h}$ in $a$ of the form

$$
p_{h}(\boldsymbol{x}, t)=p_{h}^{(0)}(\boldsymbol{x}, t)+a p_{h}^{(1)}(\boldsymbol{x}, t)+a^{2} p_{h}^{(2)}(\boldsymbol{x}, t)+\ldots,
$$

using a Neumann or Born expansion. This expansion is easily obtained by repeatedly replacing $p_{h}(\mathbf{0}, s)$ in equation (2.23) with the right hand side of equation (2.23) evaluated at $\boldsymbol{x}=\mathbf{0}$. Note that this technique leaves an explicit remainder with which we could perhaps estimate the error between the asymptotic expansion and $p_{h}(\boldsymbol{x}, t)$. For 
our purposes it suffices to just calculate the first three terms of the expansion. We find

$$
\begin{aligned}
p_{h}(\boldsymbol{x}, t)=G_{h}\left(\boldsymbol{x}-\boldsymbol{x}_{0}, t\right) & -4 \pi D a \int_{0}^{t} G_{h}(\boldsymbol{x}, t-s) G_{h}\left(\boldsymbol{x}_{0}, s\right) d s \\
& +(4 \pi D a)^{2} \int_{0}^{t} G_{h}(\boldsymbol{x}, t-s) \int_{0}^{s} G_{h}\left(\mathbf{0}, s-s^{\prime}\right) p_{h}\left(\mathbf{0}, s^{\prime}\right) d s^{\prime} d s, \\
=G_{h}\left(\boldsymbol{x}-\boldsymbol{x}_{0}, t\right) & -4 \pi D a \int_{0}^{t} G_{h}(\boldsymbol{x}, t-s) G_{h}\left(\boldsymbol{x}_{0}, s\right) d s \\
& +(4 \pi D a)^{2} \int_{0}^{t} G_{h}(\boldsymbol{x}, t-s) \int_{0}^{s} G_{h}\left(\mathbf{0}, s-s^{\prime}\right) G_{h}\left(\boldsymbol{x}_{0}, s^{\prime}\right) d s^{\prime} d s \\
& +a^{3} R_{a}(\boldsymbol{x}, t),
\end{aligned}
$$

where $a^{3} R_{a}(\boldsymbol{x}, t)$ denotes the remainder when the expansion is stopped at second order. The expansion of $(2.20)$ is then

THEOREM 2.4.

$$
\begin{aligned}
& p_{h}^{(0)}(\boldsymbol{x}, t)=G_{h}\left(\boldsymbol{x}-\boldsymbol{x}_{0}, t\right) \\
& p_{h}^{(1)}(\boldsymbol{x}, t)=-4 \pi D \int_{0}^{t} G_{h}(\boldsymbol{x}, t-s) G_{h}\left(\boldsymbol{x}_{0}, s\right) d s \\
& p_{h}^{(2)}(\boldsymbol{x}, t)=(4 \pi D)^{2} \int_{0}^{t} G_{h}(\boldsymbol{x}, t-s) \int_{0}^{s} G_{h}\left(\mathbf{0}, s-s^{\prime}\right) G_{h}\left(\boldsymbol{x}_{0}, s^{\prime}\right) d s^{\prime} d s .
\end{aligned}
$$

The formal continuum limit of $(2.25)$ is

$$
-4 \pi D \int_{0}^{t} G(\boldsymbol{x}, t-s) G\left(\boldsymbol{x}_{0}, s\right) d s .
$$

Denote this expression by $u(t)$. To find an explicit functional form of $u(t)$ we make use of the Laplace transform. Let $\tilde{f}(s)$ denote the Laplace transform of a function $f(t)$. Taking the transform of equation (2.27) in $t$, we find

$$
\begin{aligned}
\tilde{u}(s) & =\frac{-1}{4 \pi D|\boldsymbol{x}|\left|\boldsymbol{x}_{0}\right|} e^{-\left(|\boldsymbol{x}|+\left|\boldsymbol{x}_{0}\right|\right) \sqrt{s / D}} \\
& =-\frac{|\boldsymbol{x}|+\left|\boldsymbol{x}_{0}\right|}{|\boldsymbol{x}|\left|\boldsymbol{x}_{0}\right|} \tilde{G}\left(\left(|\boldsymbol{x}|+\left|\boldsymbol{x}_{0}\right|\right) \hat{\boldsymbol{x}}, s\right)
\end{aligned}
$$

where $\hat{\boldsymbol{x}}=\boldsymbol{x} /|\boldsymbol{x}|$ is a unit vector in the direction $\boldsymbol{x}$. Note that $G(|\boldsymbol{x}| \hat{\boldsymbol{x}}, t)$ is a radially symmetric function in $\boldsymbol{x}$, and therefore independent of $\hat{\boldsymbol{x}}$. Taking the inverse Laplace transform of $\tilde{u}(s)$ we find

$$
-4 \pi D \int_{0}^{t} G(\boldsymbol{x}, t-s) G\left(\boldsymbol{x}_{0}, s\right) d s=-\frac{|\boldsymbol{x}|+\left|\boldsymbol{x}_{0}\right|}{|\boldsymbol{x}|\left|\boldsymbol{x}_{0}\right|} G\left(\left(|\boldsymbol{x}|+\left|\boldsymbol{x}_{0}\right|\right) \hat{\boldsymbol{x}}, t\right) .
$$

2.2.2. Perturbation Theory for SCDLR Model. There are a number of different techniques that give the asymptotic expansion of equation (2.19) as $a \rightarrow 0$. We give the exact solution of (2.19) in Theorem 2.5 below, and show that it can be directly expanded in $a$ in Theorem 2.6. Alternatively, the first three terms of the expansion can be derived through the use of the pseudo-potential approximation (2.22) 
to the Dirichlet boundary condition in (2.19). The solution to the new diffusion equation with pseudo-potential is then itself an asymptotic approximation to the solution of (2.19), accurate through second order in $a$. This can be seen by comparing the expansion of the exact solution in Theorem 2.6 to the expansion of the pseudopotential solution, see [27].

To derive the exact solution to (2.19), we find it useful to work in spherical coordinates, and make the change of variables $\boldsymbol{x} \rightarrow(r, \theta, \phi), r \in[a, \infty), \theta \in[0, \pi)$, and $\phi \in[0,2 \pi)$. Similarly, we will let $p(r, \theta, \phi, t)=p(\boldsymbol{x}, t)$, and $\boldsymbol{x}_{0} \rightarrow\left(r_{0}, \theta_{0}, \phi_{0}\right)$.

The exact solution to (2.19) can be found using the Weber Transform [23]. Denote by $j_{l}(r)$ and $\eta_{l}(r)$ the l'th spherical Bessel functions of the first and second kind respectively, and let

$$
q_{l}(s, u)=j_{l}(s) \eta_{l}(u)-\eta_{l}(s) j_{l}(u) .
$$

The forward Weber transform of a function $f(r)$, on the interval $[a, \infty)$, is defined to be

$$
F(\lambda, a)=\sqrt{\frac{2}{\pi}} \int_{a}^{\infty} q_{l}(\lambda r, \lambda a) f(r) r^{2} d r
$$

The inverse Weber transform of $F(\lambda, a)$ is then given by

$$
f(r)=\sqrt{\frac{2}{\pi}} \int_{0}^{\infty} \frac{q_{l}(\lambda r, \lambda a)}{j_{l}^{2}(\lambda a)+\eta_{l}^{2}(\lambda a)} F(\lambda, a) \lambda^{2} d \lambda .
$$

Using the Weber transform and an expansion in Legendre polynomials, $P_{l}(\cos (\gamma))$, with $\cos (\gamma)=\cos (\theta) \cos \left(\theta_{0}\right)+\sin (\theta) \sin \left(\theta_{0}\right) \cos \left(\phi-\phi_{0}\right)$, we find

THEOREM 2.5. The solution to the freespace diffusion equation with a zero Dirichlet boundary condition on a sphere of radius a, equation (2.19), is given by

$$
p(r, \theta, \phi, t)=\sum_{l=0}^{\infty} \frac{2 l+1}{2 \pi^{2}}\left[\int_{0}^{\infty} \frac{q_{l}(\lambda r, \lambda a)}{j_{l}^{2}(\lambda a)+\eta_{l}^{2}(\lambda a)} q_{l}\left(\lambda r_{0}, \lambda a\right) e^{-\lambda^{2} D t} \lambda^{2} d \lambda\right] P_{l}(\cos (\gamma) .
$$

We again let $\hat{\boldsymbol{x}}=\boldsymbol{x} /|\boldsymbol{x}|$, so that $\hat{\boldsymbol{x}}$ is a unit vector in the same direction as $\boldsymbol{x}$. The first three terms in the expansion of $p(\boldsymbol{x}, t)$ are then given by

THEOREM 2.6. The solution (2.29) to the problem of diffusing to an absorbing sphere (2.19) has the asymptotic expansion for small a,

$$
p(\boldsymbol{x}, t) \sim p^{(0)}(\boldsymbol{x}, t)+a p^{(1)}(\boldsymbol{x}, t)+a^{2} p^{(2)}(\boldsymbol{x}, t)+\ldots,
$$

where

$$
\begin{aligned}
& p^{(0)}(\boldsymbol{x}, t)=G\left(\boldsymbol{x}-\boldsymbol{x}_{0}, t\right), \\
& p^{(1)}(\boldsymbol{x}, t)=-\frac{|\boldsymbol{x}|+\left|\boldsymbol{x}_{0}\right|}{|\boldsymbol{x}|\left|\boldsymbol{x}_{0}\right|} G\left(\left(|\boldsymbol{x}|+\left|\boldsymbol{x}_{0}\right|\right) \hat{\boldsymbol{x}}, t\right), \\
& p^{(2)}(\boldsymbol{x}, t)=\frac{2 D t-\left(|\boldsymbol{x}|+\left|\boldsymbol{x}_{0}\right|\right)^{2}}{2 D t|\boldsymbol{x}|\left|\boldsymbol{x}_{0}\right|} G\left(\left(|\boldsymbol{x}|+\left|\boldsymbol{x}_{0}\right|\right) \hat{\boldsymbol{x}}, t\right) .
\end{aligned}
$$

Proof. Notice in (2.29) that all $a$ dependence is in the bracketed term. Denoting this term by $R_{l}\left(r, r_{0}, t\right)$, we can calculate an asymptotic expansion of $R_{l}$ for small 
a. This expansion is a straightforward application of the well-known expansions of $j_{l}(\lambda a)$ [1, eq. 10.1.2] and $\eta_{l}(\lambda a)$ [1, eq. 10.1.3] for small $a$. We find, through second order in $a$, that

$$
R_{l}\left(r, r_{0}, t\right) \sim R_{l}^{(0)}\left(r, r_{0}, t\right)+a R_{l}^{(1)}\left(r, r_{0}, t\right)+a^{2} R_{l}^{(2)}\left(r, r_{0}, t\right)+\ldots
$$

where

$$
\begin{aligned}
& R_{l}^{(0)}\left(r, r_{0}, t\right)=\int_{0}^{\infty} j_{l}(\lambda r) j_{l}\left(\lambda r_{0}\right) e^{-\lambda^{2} D t} \lambda^{2} d \lambda \\
& R_{l}^{(1)}\left(r, r_{0}, t\right)=\left\{\begin{array}{l}
0, \quad l>0, \\
\int_{0}^{\infty}\left(j_{0}(\lambda r) \eta_{0}\left(\lambda r_{0}\right)+\eta_{0}(\lambda r) j_{0}\left(\lambda r_{0}\right)\right) e^{-\lambda^{2} D t} \lambda^{3} d \lambda, \quad l=0,
\end{array}\right. \\
& R_{l}^{(2)}\left(r, r_{0}, t\right)=\left\{\begin{array}{l}
0, \quad l>0, \\
\int_{0}^{\infty}\left(\eta_{0}(\lambda r) \eta_{0}\left(\lambda r_{0}\right)-j_{0}(\lambda r) j_{0}\left(\lambda r_{0}\right)\right) e^{-\lambda^{2} D t} \lambda^{4} d \lambda, \quad l=0 .
\end{array}\right.
\end{aligned}
$$

Using this expansion we may derive an expansion for $p(r, \theta, \phi, t)$. We will need several identities involving the spherical Bessel functions. Foremost is the following,

$$
\begin{aligned}
G(\boldsymbol{x}, t) & =\int_{\mathbb{R}^{3}} e^{-4 \pi^{2}|\boldsymbol{\xi}|^{2} D t} e^{2 \pi i \boldsymbol{\xi} \cdot \boldsymbol{x}} d \boldsymbol{\xi}, \\
& =\frac{1}{2 \pi^{2}} \int_{0}^{\infty} j_{0}(\lambda|\boldsymbol{x}|) e^{-\lambda^{2} D t} \lambda^{2} d \lambda .
\end{aligned}
$$

Here the first integral is the well-known Fourier representation of $G(\boldsymbol{x}, t)$. Switching $\boldsymbol{\xi}$ to spherical coordinates in the Fourier integral and performing the angular integrations gives (2.34).

Recall that $j_{0}(r)=\sin (r) / r, \eta_{0}(r)=-\cos (r) / r$, and $P_{0}(\cos (\gamma))=1$. Substituting these expressions into $R_{0}^{(1)}\left(r, r_{0}, t\right)$ and $R_{0}^{(2)}\left(r, r_{0}, t\right)$, evaluating the subsequent integrals, and using (2.34), we obtain (2.32) and (2.33). Using [1, eq. 10.1.45] and (2.34), we obtain (2.31).

2.2.3. Error Between Asymptotic Expansions of the SCDLR Model and RDME for Small $h$. We now examine the error between corresponding terms of the asymptotic expansions from Sections 2.2.1 and 2.2.2. Our main results are

Theorem 2.7. Assume $\boldsymbol{x}=h \boldsymbol{j} \neq \mathbf{0}, \boldsymbol{x}_{0}=h \boldsymbol{j}_{0} \neq \mathbf{0}$, and both are fixed as $h \rightarrow 0$. Then for all $t>0$ and $h$ sufficiently small,

$$
\begin{array}{lll}
\lim _{h \rightarrow 0} p_{h}^{(0)}(\boldsymbol{x}, t)=p^{(0)}(\boldsymbol{x}, t), & \text { with } & \left|p_{h}^{(0)}(\boldsymbol{x}, t)-p^{(0)}(\boldsymbol{x}, t)\right|=O\left(\frac{h^{2}}{t^{5 / 2}}\right), \\
\lim _{h \rightarrow 0} p_{h}^{(1)}(\boldsymbol{x}, t)=p^{(1)}(\boldsymbol{x}, t), & \text { with } & \left|p_{h}^{(1)}(\boldsymbol{x}, t)-p^{(1)}(\boldsymbol{x}, t)\right|=O\left(t h^{2-\epsilon}\right)
\end{array}
$$

where $\epsilon$ may be chosen arbitrarily small. For all fixed $t>0$,

$$
p_{h}^{(2)}(\boldsymbol{x}, t) \geq \frac{C}{h}, \quad \text { for } h \text { sufficiently small, }
$$

where $C$ is strictly positive and constant in $h$, but may depend on $t$ or $D$.

This theorem demonstrates that the reaction-diffusion master equation is a convergent asymptotic approximation to the SCDLR model only through first order in the perturbation expansion. For $h$ sufficiently small, the second order term will diverge 


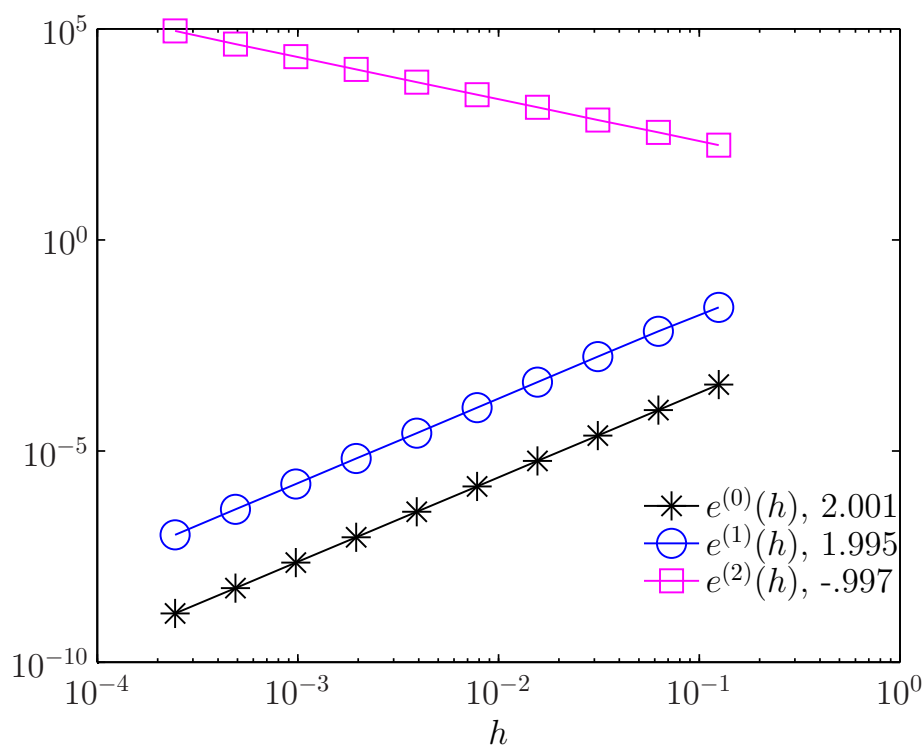

Fig. 2.3: Absolute error in asymptotic expansion terms. $e^{(i)}(h)=$ $\left|p_{h}^{(i)}(\boldsymbol{x}, t)-p^{(i)}(\boldsymbol{x}, t)\right|$, where $t=.5, \boldsymbol{x}=\boldsymbol{x}_{0}=(1 / 8,1 / 8,1 / 8)$, and $D=1$. Numbers in the inset within the figure denote the slope of the best fit line through each curve.

like $1 / h$ as $h \rightarrow 0$. The master equation model will then give a good approximation to the SCDLR model only when $h$ is small enough that the first two terms in the asymptotic expansion (2.30) are well-approximated, while $a$ is sufficiently small and $h$ sufficiently large that the divergence of higher order terms is small.

Note that the divergence of the second order term follows from the behavior as $h \rightarrow 0$ of the time integral of the continuous-time discrete-space Green's function evaluated at the origin. The proof of the theorem demonstrates that

$$
\int_{0}^{t} G_{h}(\mathbf{0}, s) d s=\frac{f_{h}(t)}{h}
$$

where, for $t$ fixed, $f_{h}(t)$ is bounded from below as $h \rightarrow 0$. The $n$ 'th term in the expansion of $p_{h}(\boldsymbol{x}, t)$ will involve $n-2$ integrals of $G_{h}(\mathbf{0}, t)$, so that we expect it to diverge like $1 / h^{n-2}$. For example, the $n=4$ term is given by

$$
p_{h}^{(3)}(\boldsymbol{x}, t)=\int_{0}^{t} G_{h}(\boldsymbol{x}, t-s) \int_{0}^{s} G_{h}\left(\mathbf{0}, s-s^{\prime}\right) \int_{0}^{s^{\prime}} G_{h}\left(\mathbf{0}, s^{\prime}, s^{\prime \prime}\right) G_{h}\left(\boldsymbol{x}_{0}, s^{\prime \prime}\right) d s^{\prime \prime} d s^{\prime} d s
$$

which we would expect to diverge like $1 / h^{2}$. Since the coefficient of the $n$ 'th term in the expansion is $a^{n-1}$, we expect the $n$ 'th term to behave like $a^{n-1} / h^{n-2}$. For $n$ large this suggests that the heuristic assumption that $h \gg k / 4 \pi D=a$ from Section 1.1.2 is a reasonable rule of thumb for choosing the mesh size.

Figure 2.3 shows the pointwise error in each of the first three terms of the asymptotic expansion as a function of $h$, for fixed $t, \boldsymbol{x}$, and $\boldsymbol{x}_{0}$. Note that for each term the 


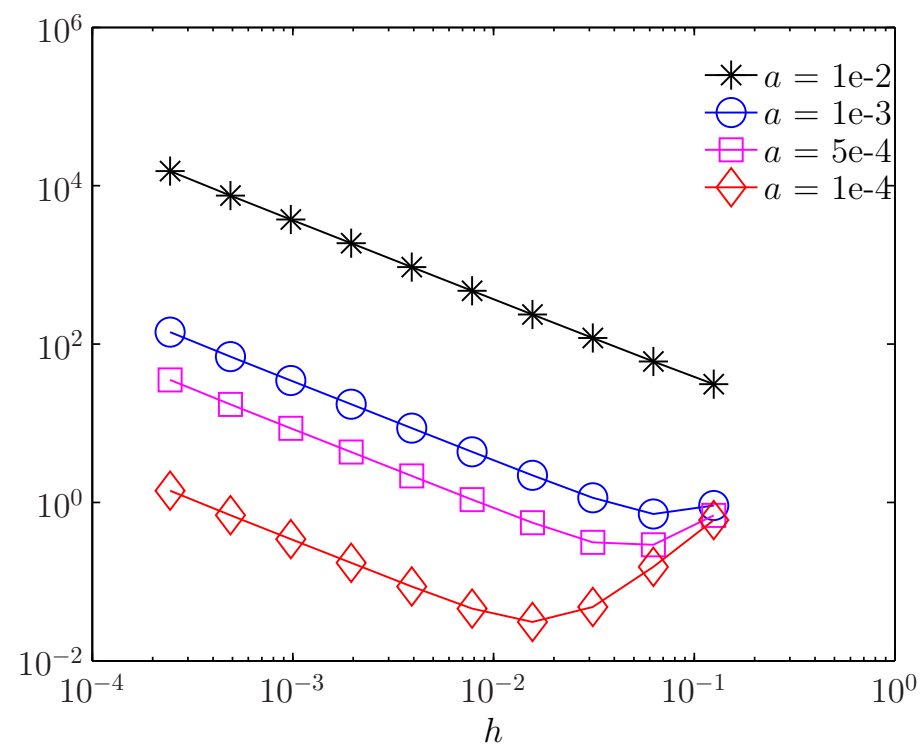

Fig. 2.4: Percent relative error in perturbation expansions through 2'nd order. Each curve plots $E_{\mathrm{REL}}(\boldsymbol{x}, t, h, a)$ vs. $h$ for different values of the reaction radius, $a$. For all curves $t=.5, \boldsymbol{x}=\boldsymbol{x}_{0}=(1 / 8,1 / 8,1 / 8)$, and $D=1$.

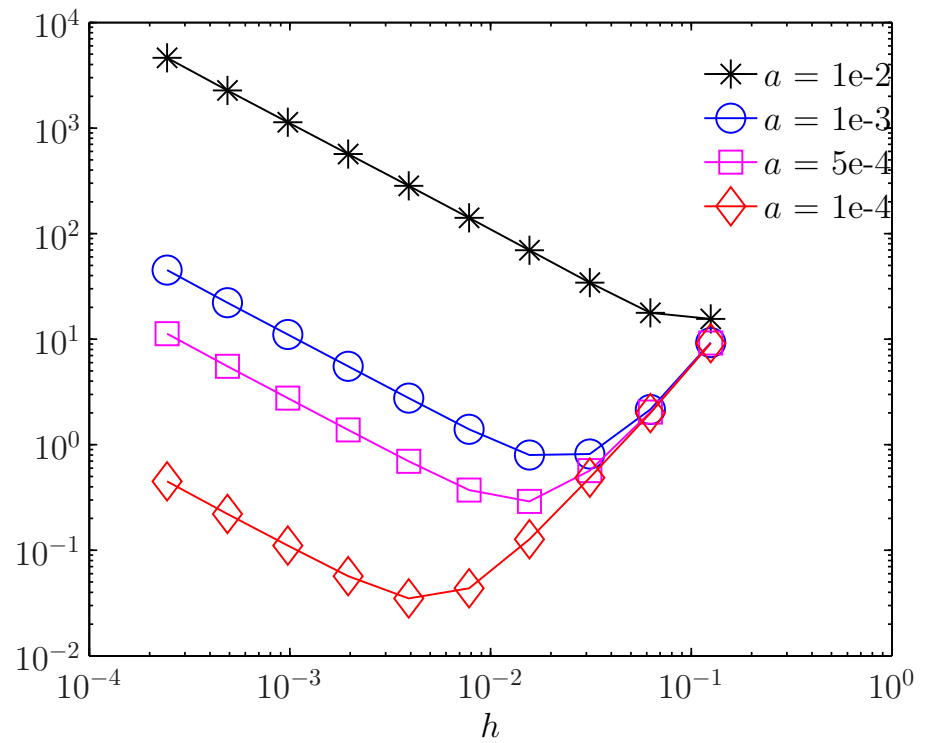

Fig. 2.5: Percent relative error in perturbation expansions through 2'nd order. Each curve plots $E_{\mathrm{REL}}(\boldsymbol{x}, t, h, a)$ vs. $h$ for different values of the reaction radius, $a$. For all curves $t=.038147, \boldsymbol{x}=\boldsymbol{x}_{0}=(1 / 8,1 / 8,1 / 8)$, and $D=1$. 
observed numerical convergence (divergence for the second order term) rate agrees with that in Theorem 2.7. Let

$$
R_{h}(\boldsymbol{x}, t, h, a)=p_{h}^{(0)}(\boldsymbol{x}, t)+a p_{h}^{(1)}(\boldsymbol{x}, t)+a^{2} p_{h}^{(2)}(\boldsymbol{x}, t),
$$

and

$$
R(\boldsymbol{x}, t, h, a)=p^{(0)}(\boldsymbol{x}, t)+a p^{(1)}(\boldsymbol{x}, t)+a^{2} p^{(2)}(\boldsymbol{x}, t) .
$$

Figures 2.4 and 2.5 plot the percent relative error between $R_{h}$ and $R$,

$$
E_{\mathrm{REL}}(\boldsymbol{x}, t, h, a)=100 \frac{R_{h}(\boldsymbol{x}, t, h, a)-R(\boldsymbol{x}, t, h, a)}{R(\boldsymbol{x}, t, h, a)},
$$

which also represents the percent relative error between the perturbation expansions of $p_{h}(\boldsymbol{x}, t)$ and $p(\boldsymbol{x}, t)$, the solution to the SCDLR model, truncated after the third term. Notice that for larger values of $h$ the relative error decreases as $h$ decreases, but that as $h$ becomes smaller the $1 / h$ divergence of the second order term begins to dominate and cause $E_{\mathrm{REL}}$ to diverge. Both Figures 2.3 and 2.4 are shown for relatively large $t$ values. Figure 2.5 shows the behavior of $E_{\mathrm{REL}}$ at a shorter time, when both $p_{h}(\boldsymbol{x}, t)$ and $p(\boldsymbol{x}, t)$ have relaxed less. The details of the numerical methods used in calculating the terms of the asymptotic expansions are explained in Appendix B.

For $a \leq 10^{-3}$ the overall relative error can be reduced below one percent. In physical units, appropriate for considering chemical systems at the scale of a eukaryotic cell, $D$ would have units of square micrometers per second, $t$ units of seconds, and $\boldsymbol{x}, \boldsymbol{x}_{0}$, and $a$ units of micrometers. This suggests that for physical reaction-radii of one nanometer or less the reaction-diffusion master equation may be a good approximation to a diffusion limited reaction. While physical reaction-radii have not been experientially determined for most biological reactions, it has been found experimentally that the LexA DNA binding protein has a physical binding potential of width $\sim 5 \AA[30]$. We caution, however, that these results are only valid for the truncated perturbation expansions, and do not necessarily hold for the error between the exact solutions $p_{h}(\boldsymbol{x}, t)$ and $p(\boldsymbol{x}, t)$. Moreover, for realistic biophysical systems, one would frequently be interested in volumes where more than one of each substrate is present; a case we have not examined herein.

Proof. [Proof of Theorem 2.7] The validity of equation (2.35) has already been established in Theorem A.1. Lemma 2.2 and Corollary A.2 imply that for all $h$ and $\epsilon$ sufficiently small, and all $t \geq 0$,

$$
\sup _{t \in[0, \infty)}\left|G_{h}(\boldsymbol{x}, t-s) G_{h}\left(\boldsymbol{x}_{0}, s\right)-G(\boldsymbol{x}, t-s) G\left(\boldsymbol{x}_{0}, s\right)\right| \leq C h^{2-\epsilon}
$$

with $C$ independent of $t, s$, and $h$. Recalling (2.28), (2.32), and (2.25) we find

$$
\left|p_{h}^{(1)}(\boldsymbol{x}, t)-p^{(1)}(\boldsymbol{x}, t)\right| \leq C t h^{2-\epsilon},
$$

which proves (2.36). We now consider the divergence of $p_{h}^{(2)}(\boldsymbol{x}, t)$. The non-negativity of $G_{h}(\boldsymbol{x}, t)$ for all $\boldsymbol{x}$ and $t \geq 0$ implies that for all $t>3 \delta>0$,

$$
p_{h}^{(2)}(\boldsymbol{x}, t) \geq(4 \pi D)^{2} \int_{2 \delta}^{t-\delta} G_{h}(\boldsymbol{x}, t-s) \int_{s-\delta}^{s} G_{h}\left(\mathbf{0}, s-s^{\prime}\right) G_{h}\left(\boldsymbol{x}_{0}, s^{\prime}\right) d s^{\prime} d s .
$$


We subsequently denote by $C$ a generic positive constant independent of $h$, but dependent on $t$. Note that $G(\boldsymbol{x}, t)$ is positive for all $\boldsymbol{x}$ and all $t>0$. Uniform convergence in time of $G_{h}(\boldsymbol{x}, t)$ to $G(\boldsymbol{x}, t)$ (Theorem A.1) implies that $\epsilon$ and $h$ may be taken sufficiently small so that

$$
\begin{aligned}
\inf _{s \in(2 \delta, t-\delta)} G_{h}(\boldsymbol{x}, t-s) & \geq \inf _{s \in(2 \delta, t-\delta)} G(\boldsymbol{x}, t-s)-\epsilon \\
& \geq C>0,
\end{aligned}
$$

and similarly

$$
\inf _{s^{\prime} \in(\delta, t-\delta)} G_{h}\left(\boldsymbol{x}_{0}, s^{\prime}\right) \geq C>0 .
$$

We then find that for all $h$ sufficiently small,

$$
\begin{aligned}
p_{h}^{(2)}(\boldsymbol{x}, t) & \geq C \int_{2 \delta}^{t-\delta} \int_{s-\delta}^{s} G_{h}\left(\mathbf{0}, s-s^{\prime}\right) d s^{\prime} d s, \\
& \geq C \int_{0}^{\delta} G_{h}(\mathbf{0}, s) d s .
\end{aligned}
$$

$G_{h}(\mathbf{0}, s)$ has the Fourier representation

$$
G_{h}(\mathbf{0}, s)=\frac{1}{h^{3}} \iiint_{\left[\frac{-1}{2} \ldots \frac{1}{2}\right]^{3}} e^{-4 D s \sum_{k=1}^{3} \sin ^{2}\left(\pi y_{k}\right) / h^{2}} d \boldsymbol{y} .
$$

As the integrand in the above integral is non-negative, we may apply Fubini's Theorem to switch the order of integration in (2.39). We find

$$
p_{h}^{(2)}(\boldsymbol{x}, t) \geq \frac{C}{h} \iiint_{\left[\frac{-1}{2} \ldots \frac{1}{2}\right]^{3}} \frac{1}{\sum_{k=1}^{3} \sin ^{2}\left(\pi y_{k}\right)}\left(1-e^{-4 D \delta \sum_{k=1}^{3} \sin ^{2}\left(\pi y_{k}\right) / h^{2}}\right) d \boldsymbol{y} .
$$

Switching to spherical coordinates in the integral we have that

$$
\begin{aligned}
p_{h}^{(2)}(\boldsymbol{x}, t) & \geq \frac{C}{h} \int_{0}^{1 / 2}\left(1-e^{-16 D \delta r^{2} / h^{2}}\right) d r, \\
& =\frac{C}{h}\left(\frac{1}{2}-\sqrt{\frac{\pi}{t}} \frac{h}{8} \operatorname{erf}\left(\frac{2 \sqrt{t}}{h}\right)\right) .
\end{aligned}
$$

Here we have used that $\pi y \geq \sin (\pi y) \geq 2 y$ on $[0,1 / 2]$. The last term in parenthesis in equation (2.40) approaches zero as $h \rightarrow 0$ and therefore

$$
p_{h}^{(2)}(\boldsymbol{x}, t) \geq \frac{C}{h},
$$

for $h$ sufficiently small, with $C$ strictly positive.

3. Conclusions. We have shown that as the mesh spacing approaches zero in the reaction-diffusion master equation model, particles undergoing a bimolecular reaction never react, and simply diffuse. In contrast, the relative errors of the truncated asymptotic expansions shown in Figures 2.4 and 2.5 suggest that for physically reasonable parameters values, the mesh spacing in the reaction-diffusion master equation 
may be chosen to give a good approximation of a spatially-continuous diffusion limited reaction model. Notice in Figure 2.5 that the mesh spacing for the minimal relative error is generally more than a factor of ten larger than the reaction-radius. This suggests that choosing the mesh spacing to satisfy the physically derived lower bound, equation (1.6), may be a good rule of thumb. Note, however, that good agreement between the truncated asymptotic expansions does not necessarily guarantee good agreement of the actual solutions of the two models. We hope to report on the error between the solutions to the reaction-diffusion master equation and the SCDLR model, for biologically relevant parameter values, in future work. Towards that end, we would like to examine this error in a more biologically relevant domain. (The restriction to $\mathbb{R}^{3}$ in the current work was made to simplify the mathematical analysis.)

The results of Section 2.2.3 suggest a means by which to improve the accuracy of the reaction-diffusion master equation as an approximation to a diffusion limited reaction; modifying/renormalizing the bimolecular reaction rate, $k / h^{3}$, so that the second order term in the asymptotic expansion of the solution to the reaction-diffusion master equation converges to the corresponding term in the asymptotic expansion of the SCDLR model. Note, this may require changing the discrete bimolecular reaction operator to couple neighboring voxels, and would presumably correspond to modifying it to converge to a pseudo-potential reaction operator like that in equation (2.22).

Finally, we would like to point out that it should be an easy modification to extend the results of this work to $\mathbb{R}^{d}$, for all $d \geq 2$. In particular, it appears that the second order term in the asymptotic expansion of the reaction-diffusion master equation diverges like $\log (h)$ in two-dimensions, and $1 / h^{d-2}$ in $d$-dimensions with $d>2$. In one-dimension the solution of the continuum model, equation (2.21), is well-defined, and we expect the solution to the reaction-diffusion master equation to converge to it.

4. Acknowledgments. SAI is supported by an NSF RTG postdoctoral fellowship. Numerical simulations that stimulated this work made use of supercomputer time on the NCSA Teragrid system awarded under grant DMS040022.

The author would like to thank several individuals for helpful discussions. Charles Peskin helped stimulate this work through discussions on the nature of the continuum limit of the reaction-diffusion master equation. In particular, he showed by Laplace transform methods that the mean binding time for a bimolecular reaction in the RDME with periodic geometry becomes infinite as the mesh spacing approaches zero, suggesting the approach of Section 2.1. David Isaacson aided the author in developing an alternative derivation of the asymptotic expansion of the solution to the SCDLR model through the use of pseudo-potentials, see [27]. Both David Isaacson and Charles Peskin provided critical comments on this manuscript. Firas Rassoul-Agha suggested both the time splitting used in the uniform convergence proof of Theorem A.1, and the bounding by an exponential trick used in the short time part of the proof. He also provided a separate proof of the uniform convergence for large times through a different argument, based on extending the local central limit theorem for discretetime random walks to continuous-time random walks.

Finally, we would like to thank Bob Guy, James P. Keener, and Eric VandenEijnden for helpful discussions.

Appendix A. Convergence of the Green's Function for the DiscreteSpace Continuous-Time Diffusion Equation. We prove the following convergence theorem 
Theorem A.1. Let $\boldsymbol{x}_{\boldsymbol{j}}=h \boldsymbol{j}$ remain fixed as $h \rightarrow 0$. Then for all $\boldsymbol{x}_{\boldsymbol{j}}$, all $t \geq \delta>0$ with $\delta$ fixed, and $h>0$ sufficiently small,

$$
\left|G_{h}\left(\boldsymbol{x}_{\boldsymbol{j}}, t\right)-G\left(\boldsymbol{x}_{\boldsymbol{j}}, t\right)\right| \leq C \frac{h^{2}}{\delta^{5 / 2}} .
$$

Here $C$ is independent of $t, h$, and $\boldsymbol{x}_{\boldsymbol{j}}$.

In addition, for $\boldsymbol{x}_{\boldsymbol{j}}$ fixed as $h \rightarrow 0$ and $\boldsymbol{x}_{\boldsymbol{j}} \neq \mathbf{0}, G_{h}\left(\boldsymbol{x}_{\boldsymbol{j}}, t\right) \rightarrow G\left(\boldsymbol{x}_{\boldsymbol{j}}, t\right)$ uniformly in all $t \geq 0$ as $h \rightarrow 0$.

Proof. We begin by proving equation (A.1). $G_{h}$ has the representation

$$
G_{h}\left(\boldsymbol{x}_{\boldsymbol{j}}, t\right)=\iiint_{\left[\frac{-1}{2 h} \cdots \frac{1}{2 h}\right]^{3}} e^{-4 D t \sum_{k=1}^{3} \frac{\sin ^{2}\left(\pi h \boldsymbol{\xi}_{k}\right)}{h^{2}}} e^{2 \pi i\left(\boldsymbol{x}_{\boldsymbol{j}}, \boldsymbol{\xi}\right)} d \boldsymbol{\xi} .
$$

Similarly,

$$
G\left(\boldsymbol{x}_{\boldsymbol{j}}, t\right)=\iiint_{\mathbb{R}^{3}} e^{-4 D t \pi^{2}|\boldsymbol{\xi}|^{2}} e^{2 \pi i\left(\boldsymbol{x}_{j}, \boldsymbol{\xi}\right)} d \boldsymbol{\xi}
$$

We find

$$
\begin{aligned}
\left|G_{h}\left(\boldsymbol{x}_{\boldsymbol{j}}, t\right)-G\left(\boldsymbol{x}_{\boldsymbol{j}}, t\right)\right| \leq & \iiint_{\left[\frac{-1}{2 h} \cdots \frac{1}{2 h}\right]^{3}}\left|e^{-4 D t \sum_{k=1}^{3} \frac{\sin ^{2}\left(\pi h \boldsymbol{\xi}_{k}\right)}{h^{2}}}-e^{-4 D t \pi^{2}|\boldsymbol{\xi}|^{2}}\right| d \boldsymbol{\xi} \\
& +\iiint_{R^{3}-\left[\frac{-1}{2 h} \cdots \frac{1}{2 h}\right]^{3}} e^{-4 D t \pi^{2}|\boldsymbol{\xi}|^{2}} d \boldsymbol{\xi} .
\end{aligned}
$$

Denote these last two integrals by $I$ and $I I$ respectively. The second integral may be bounded by expanding the domain of integration to the exterior of the sphere of radius $1 / 2 h$. Switching to polar coordinates this gives

$$
\begin{aligned}
I I & \leq 4 \pi \int_{\frac{1}{2 h}}^{\infty} r^{2} e^{-4 D t \pi^{2} r^{2}} d r, \\
& =\frac{1}{4 \pi D t h} e^{-\pi^{2} D t / h^{2}}+\frac{1}{8(\pi D t)^{3 / 2}} \operatorname{erfc}\left(\frac{\pi \sqrt{D t}}{h}\right) .
\end{aligned}
$$

Using that [1, eq. 7.1.13]

$$
\operatorname{erfc}(r) \leq e^{-r^{2}}, \quad r \geq 0,
$$

we find

$$
I I \leq \frac{1}{h(4 \pi D t)^{3 / 2}}(2 \sqrt{\pi D t}+h) e^{-\pi^{2} D t / h^{2}}, \quad \forall t>0 .
$$

For $h$ sufficiently small this error bound will satisfy (A.1).

To bound $I$, we begin by Taylor expanding the first term of the integrand in $I$ about the point $\pi h \boldsymbol{\xi}$. Note that $\pi h \boldsymbol{\xi} \in[-\pi / 2, \pi / 2]^{3}$ even as $h$ changes. Let, $\boldsymbol{y}=\pi h \boldsymbol{\xi}$ and define

$$
f(\boldsymbol{y})=4 D t \sum_{k=1}^{3} \frac{\sin ^{2}\left(y_{k}\right) \pi^{2} \boldsymbol{\xi}_{k}^{2}}{\boldsymbol{y}_{k}^{2}}
$$


$e^{-f(\boldsymbol{y})}$ has the two-term Taylor expansion with remainder

$$
e^{-f(\boldsymbol{y})}=e^{-4 D t \pi^{2}|\boldsymbol{\xi}|^{2}}+\frac{1}{2}\left(\boldsymbol{y}, D^{2} e^{-f(\overline{\boldsymbol{y}})} \boldsymbol{y}\right), \quad \overline{\boldsymbol{y}} \in\left[-\frac{1}{2}, \frac{1}{2}\right]^{3} .
$$

Here $D^{2}$ denotes the matrix of second derivatives of $f(\boldsymbol{y})$, and the first derivative term disappears since the gradient of $f(\boldsymbol{y})$ is zero at $\boldsymbol{y}=\mathbf{0}$. The second derivative term is given by

$$
\left(D^{2} e^{-f(\boldsymbol{y})}\right)_{i, j}=-\left(\frac{\partial^{2} f}{\partial y_{i} \partial y_{j}}(\boldsymbol{y})-\frac{\partial f}{\partial y_{i}}(\boldsymbol{y}) \frac{\partial f}{\partial y_{j}}(\boldsymbol{y})\right) e^{-f(\boldsymbol{y})},
$$

where

$$
\frac{\partial f}{\partial y_{i}}(\boldsymbol{y})=-4 D t \pi^{2} \xi_{k}^{2}\left(\frac{2 \sin ^{2}\left(y_{i}\right)}{y_{i}^{3}}-\frac{\sin \left(2 y_{i}\right)}{y_{i}^{2}}\right)
$$

and

$$
\frac{\partial^{2} f}{\partial y_{i} \partial y_{j}}(\boldsymbol{y})=\left\{\begin{array}{l}
0, \quad i \neq j, \\
4 D t \pi^{2} \xi_{i}^{2}\left(2 y_{i}^{2} \cos \left(2 y_{i}\right)-4 y_{i} \sin \left(2 y_{i}\right)+6 \sin ^{2}\left(y_{i}\right)\right) / y_{i}^{4} .
\end{array}\right.
$$

Since $\bar{y}_{i} \leq 1 / 2$, we may uniformly bound in $\boldsymbol{y}$ the remainders for the one term Taylor expansions of the derivatives of $f(\overline{\boldsymbol{y}})$. We find

$$
D^{2} e^{-f(\overline{\boldsymbol{y}})} \leq e^{-f(\overline{\boldsymbol{y}})} A(\boldsymbol{\xi}, t),
$$

where

$$
A_{i, j}(\boldsymbol{\xi}, t)=\left\{\begin{array}{l}
O\left(t^{2} \xi_{i}^{2} \xi_{j}^{2}\right), \quad i \neq j \\
O\left(t^{2} \xi_{i}^{4}+t \xi_{i}^{2}\right), \quad i=j
\end{array}\right.
$$

Letting $\|\cdot\|_{2}$ denote the matrix norm induced by the Euclidean vector norm, this estimate gives the bound

$$
\begin{aligned}
\left(\boldsymbol{y}, D^{2} e^{-f(\overline{\boldsymbol{y}})} \boldsymbol{y}\right) & \leq e^{-f(\overline{\boldsymbol{y}})}\|A(\boldsymbol{\xi}, t)\|_{2}|\boldsymbol{y}|^{2} \\
& \leq C e^{-f(\overline{\boldsymbol{y}})}\|A(\boldsymbol{\xi}, t)\|_{F}|\boldsymbol{\xi}|^{2} h^{2},
\end{aligned}
$$

where $\|\cdot\|_{F}$ denotes the matrix Frobenius norm. Let $M_{n}(\boldsymbol{\xi})$ be a three-dimensional monomial of degree $n$, we have

$$
\begin{aligned}
\|A(\boldsymbol{\xi}, t)\|_{F} & =\left(O\left(t^{4} M_{8}(\boldsymbol{\xi})\right)+O\left(t^{2} M_{4}(\boldsymbol{\xi})\right)\right)^{\frac{1}{2}} \\
& \leq O\left(t^{2}|\boldsymbol{\xi}|^{4}\right)+O\left(t|\boldsymbol{\xi}|^{2}\right)
\end{aligned}
$$

for specific monomials $M_{8}(\boldsymbol{\xi})$ and $M_{4}(\boldsymbol{\xi})$. (This follows since $M_{2 n}(\boldsymbol{\xi}) \leq C|\boldsymbol{\xi}|^{2 n}$ for all $n)$. Moreover, since

$$
\sin ^{2}(x) \geq \frac{4}{\pi^{2}} x^{2}, \quad \forall x \in\left[-\frac{\pi}{2}, \frac{\pi}{2}\right]
$$

we have that

$$
e^{-f(\overline{\boldsymbol{y}})} \leq e^{-16 D t|\boldsymbol{\xi}|^{2}} .
$$


Combining the two preceding estimates, (A.6) and (A.7), with equation (A.5) we find

$$
\left(\boldsymbol{y}, D^{2} e^{-f(\overline{\boldsymbol{y}})} \boldsymbol{y}\right) \leq\left(O\left(t^{2}|\boldsymbol{\xi}|^{6}\right)+O\left(t|\boldsymbol{\xi}|^{4}\right)\right) e^{-16 D t|\boldsymbol{\xi}|^{2}} h^{2} .
$$

This estimate implies that

$$
\begin{aligned}
I & \leq h^{2} \iiint_{\left[\frac{-1}{2 h} \ldots \frac{1}{2 h}\right]^{3}}\left(O\left(t^{2}|\boldsymbol{\xi}|^{6}\right)+O\left(t|\boldsymbol{\xi}|^{4}\right)\right) e^{-16 D t|\boldsymbol{\xi}|^{2}} d \boldsymbol{\xi} \\
& \leq h^{2} \int_{0}^{\infty}\left(O\left(t^{2} r^{8}\right)+O\left(t r^{6}\right)\right) e^{-16 D t r^{2}} d r, \\
& =O\left(\frac{h^{2}}{t^{5 / 2}}\right) .
\end{aligned}
$$

For $t \geq \delta$ the desired bound in equation (A.1) follows.

We now prove the second assertion of the theorem, that for $\boldsymbol{x}_{\boldsymbol{j}} \neq \mathbf{0}$ and fixed as $h \rightarrow 0, G_{h}\left(\boldsymbol{x}_{\boldsymbol{j}}, t\right) \rightarrow G\left(\boldsymbol{x}_{\boldsymbol{j}}, t\right)$ as $h \rightarrow 0$ uniformly in all $t \geq 0$. To prove the assertion, we find it necessary to treat separately very short and all other times. Let $a_{h}=h^{-1-\mu}$ with $\mu \in(0,1)$, so that $a_{h} h^{2} \rightarrow 0$ and $a_{h} h \rightarrow \infty$ as $h \rightarrow 0$. The condition $a_{h} h \rightarrow \infty$ as $h \rightarrow 0$ will turn out to be necessary to prove uniform convergence for short times. We wish to show that

$$
\lim _{h \rightarrow 0} \sup _{t \in[0, \infty)}\left|G_{h}\left(\boldsymbol{x}_{\boldsymbol{j}}, t\right)-G\left(\boldsymbol{x}_{\boldsymbol{j}}, t\right)\right|=0 .
$$

This is equivalent to proving that for any $\epsilon>0$ and all $h$ sufficiently small

$$
\sup _{t \in\left[0, a_{h} h^{2}\right)}\left|G_{h}\left(\boldsymbol{x}_{\boldsymbol{j}}, t\right)-G\left(\boldsymbol{x}_{\boldsymbol{j}}, t\right)\right|<\epsilon,
$$

and

$$
\sup _{t \in\left[a_{h} h^{2}, \infty\right)}\left|G_{h}\left(\boldsymbol{x}_{\boldsymbol{j}}, t\right)-G\left(\boldsymbol{x}_{\boldsymbol{j}}, t\right)\right|<\epsilon .
$$

We begin by proving (A.10). Equation (A.2) bounds the error for fixed $t$ by two terms, $I$ and $I I$, with $I I$ satisfying equation (A.4). Let $0<R<1 /(2 h)$. $I$ satisfies

$$
\begin{aligned}
I \leq & \iiint_{|\boldsymbol{\xi}|<R}\left|e^{-4 D t \sum_{k=1}^{3} \frac{\sin ^{2}\left(\pi h \xi_{k}\right)}{h^{2}}}-e^{-4 D t \pi^{2}|\boldsymbol{\xi}|^{2}}\right| d \boldsymbol{\xi}, \\
& +\iiint_{\left[\frac{-1}{2 h} \cdots \frac{1}{2 h}\right]^{3}-\{|\boldsymbol{\xi}|<R\}}\left|e^{-4 D t \sum_{k=1}^{3} \frac{\sin ^{2}\left(\pi h \boldsymbol{\xi}_{k}\right)}{h^{2}}}-e^{-4 D t \pi^{2}|\boldsymbol{\xi}|^{2}}\right| d \boldsymbol{\xi} .
\end{aligned}
$$

We subsequently label the two terms on the right hand side by $I_{a}$ and $I_{b}$ respectively. In what follows, $C$ will denote an arbitrary constant, independent of $h$ and $t$. The argument giving equation (A.8) holds for $I_{a}$, and shows that

$$
\begin{aligned}
I_{a} & \leq C h^{2} \iiint_{|\boldsymbol{\xi}|<R}\left(t^{2}|\boldsymbol{\xi}|^{6}+t|\boldsymbol{\xi}|^{4}\right) e^{-16 D t|\boldsymbol{\xi}|^{2}} d \boldsymbol{\xi}, \\
& \leq C h^{2} R^{3} \iiint_{|\boldsymbol{\xi}|<R}\left(t^{2}|\boldsymbol{\xi}|^{3}+t|\boldsymbol{\xi}|\right) e^{-16 D t|\boldsymbol{\xi}|^{2}} d \boldsymbol{\xi}, \\
& =C \frac{h^{2} R^{3}}{t}, \quad \forall t>0 .
\end{aligned}
$$


Using (A.7), we have that

$$
\begin{aligned}
I_{b} & \leq C \int_{R}^{\infty} r^{2} e^{-16 D t r^{2}} d r \\
& =\frac{C}{t^{3 / 2}}\left[8 R \sqrt{t} e^{-16 D t R^{2}}+\sqrt{\pi} \operatorname{erfc}(4 R \sqrt{D t})\right],
\end{aligned}
$$

which by (A.3) implies

$$
I_{b} \leq \frac{C}{t^{3 / 2}}[8 R \sqrt{t}+\sqrt{\pi}] e^{-16 D t R^{2}}, \quad \forall t>0 .
$$

To summarize, we have shown that

$$
\left|G_{h}\left(\boldsymbol{x}_{\boldsymbol{j}}, t\right)-G\left(\boldsymbol{x}_{\boldsymbol{j}}, t\right)\right| \leq I_{a}+I_{b}+I I
$$

where

$$
\begin{aligned}
& I_{a} \leq C \frac{h^{2} R^{3}}{t}, \\
& I_{b} \leq \frac{C}{t^{3 / 2}}[8 R \sqrt{t}+\sqrt{\pi}] e^{-16 D t R^{2}}, \\
& I I \leq \frac{1}{h(8 \pi D t)^{3 / 2}}(2 \sqrt{\pi D t}+h) e^{-\pi^{2} D t / h^{2}} .
\end{aligned}
$$

We now show this error can be made uniformly small in $t$ for $t \geq a_{h} h^{2}$. Substituting this inequality into equation (A.13) we find

$$
\begin{aligned}
I_{a} & \leq C \frac{R^{3}}{a_{h}}, \\
I_{b} & \leq \frac{C}{\left(a_{h} h^{2}\right)^{3 / 2}}\left[8 R \sqrt{a_{h}} h+\sqrt{\pi}\right] e^{-16 D a_{h} h^{2} R^{2}}, \\
I I & \leq \frac{1}{\left(8 \pi D a_{h} h^{2}\right)^{3 / 2}}\left(2 \sqrt{\pi D a_{h}}+1\right) e^{-\pi^{2} D a_{h}} .
\end{aligned}
$$

Clearly $I I$ will be arbitrarily small for all $h$ sufficiently small, so it remains to show that $R$ and $a_{h}$ can be chosen such that $I_{a}$ and $I_{b}$ approach zero as $h \rightarrow 0$. This will hold if

$$
\begin{aligned}
\lim _{h \rightarrow 0} \frac{R^{3}}{a_{h}} & =0, \\
\lim _{h \rightarrow 0} a_{h} h^{2} R^{2} & =\infty,
\end{aligned}
$$

with $0<R<1 /(2 h), a_{h} h \rightarrow \infty$ as $h \rightarrow 0$, and $a_{h} h^{2} \rightarrow 0$ as $h \rightarrow 0$. As mentioned earlier, we let $a_{h}=h^{-1-\mu}$ with $\mu \in(0,1)$. In addition, let $R=h^{-\alpha} / 2$ with $\alpha \in$ $(0,1)$. Note that this choice of $\alpha$ allows $0<R<1 /(2 h)$ for $h$ small as required. Equation (A.15) then holds if

$$
\begin{aligned}
& 1+\mu-3 \alpha>0 \\
& 2 \alpha+\mu-1>0
\end{aligned}
$$

These equations have an infinite number of valid solutions which also satisfy the other necessary conditions on $R$ and $a_{h}$. For example, $\alpha=1 / 4$ and $\mu=3 / 4$. We have therefore shown that equation (A.10) holds. 
We now prove that equation (A.9) holds. We denote the first non-zero component of $\boldsymbol{x}_{\boldsymbol{j}}$ by $x$. Note that $G_{h}\left(\boldsymbol{x}_{\boldsymbol{j}}, t\right)$ may be written in terms of the solution to the onedimensional continuous-time discrete-space diffusion equation, $g_{h}\left(x_{j_{k}}, t\right)$, as

$$
G_{h}\left(\boldsymbol{x}_{\boldsymbol{j}}, t\right)=\prod_{k=1}^{3} g_{h}\left(x_{j_{k}}, t\right)
$$

Non-negativity of $g_{h}\left(x_{j_{k}}, t\right)$ and the conservation relation

$$
\sum_{n=-\infty}^{\infty} g_{h}(n h, t) h=1
$$

imply

$$
G_{h}\left(\boldsymbol{x}_{\boldsymbol{j}}, t\right) \leq \frac{1}{h^{2}} g_{h}(x, t) .
$$

Without loss of generality, we now assume that $x>0$. Then for any positive number, $\lambda$,

$$
\frac{1}{h^{2}} g_{h}(x, t) \leq \frac{1}{h^{2}} \sum_{n=-\infty}^{\infty} e^{\lambda n-\lambda x / h} g_{h}(n h, t)
$$

We define

$$
M(\lambda, t)=\sum_{n=-\infty}^{\infty} e^{\lambda n} g_{h}(n h, t)
$$

Note that $g_{h}(n h, t) h$, is the probability distribution for a continuous-time random walk in $\mathbb{R}^{1}$ with nearest-neighbor transition rate $D / h^{2}$ and lattice spacing $h$. Likewise, $M(\lambda, t) h$ is the moment generating function associated with $g_{h}(n h, t) h$. Differentiating $M(\lambda, t)$ and using that $g_{h}(n h, t)$ satisfies the continuous-time discrete-space diffusion equation, we find

$$
\frac{d M}{d t}(\lambda, t)=\frac{2 D}{h^{2}}(\cosh (\lambda)-1) M(\lambda, t) .
$$

As $g_{h}(n h, 0)=\delta_{n 0} / h$, we have $M(\lambda, 0)=1 / h$. This implies

$$
\begin{aligned}
M(\lambda, t) & =\frac{1}{h} e^{(\cosh (\lambda)-1)\left(2 D t / h^{2}\right)}, \\
& \leq \frac{1}{h} e^{(\cosh (\lambda)-1)\left(2 D a_{h}\right)},
\end{aligned}
$$

so that

$$
\frac{1}{h^{2}} g_{h}(x, t) \leq \frac{1}{h^{3}} e^{-\lambda x / h} e^{(\cosh (\lambda)-1)\left(2 D a_{h}\right)} .
$$

Since $\lambda$ is arbitrary, we now assume that $\lambda$ is small. We may then expand the $\cosh (\lambda)$ term so that

$$
\frac{1}{h^{2}} g_{h}(x, t) \leq \frac{1}{h^{3}} e^{-\lambda x / h} e^{D a_{h} \lambda^{2}} e^{O\left(\lambda^{4} a_{h}\right)}
$$


Choosing $\lambda=x / 2 D a_{h} h$, which will be small for $h$ sufficiently small, we find

$$
\frac{1}{h^{2}} g_{h}(x, t) \leq \frac{1}{h^{3}} e^{-x^{2} / 2 D a_{h} h^{2}} e^{O\left(1 / a_{h}^{3} h^{4}\right)} .
$$

Since $a_{h}=h^{-1-\mu}, \mu \in(0,1)$, we see that the last exponential will approach 1 as $h \rightarrow 0$ if $\mu>1 / 3$. Recall that $\mu$ must also satisfy the two inequalities given in (A.16). The choice of $\mu=3 / 4$ given earlier satisfies all required inequalities. We have therefore shown that for all $h$ sufficiently small,

$$
G_{h}\left(\boldsymbol{x}_{\boldsymbol{j}}, t\right) \leq \frac{C}{h^{3}} e^{-x^{2} / 4 D a_{h} h^{2}}, \quad \forall t \in\left[0, a_{h} h^{2}\right),
$$

which proves (A.9) and completes the proof of uniform convergence in time.

Corollary A.2. Let $\boldsymbol{x}_{\boldsymbol{j}}=\boldsymbol{j} h$ be fixed as $h \rightarrow 0$, and $\boldsymbol{x}_{\boldsymbol{j}} \neq \mathbf{0}$. Then for all $h$ sufficiently small, and any $\epsilon>0$ sufficiently small,

$$
\sup _{t \in[0, \infty)}\left|G_{h}\left(\boldsymbol{x}_{\boldsymbol{j}}, t\right)-G\left(\boldsymbol{x}_{\boldsymbol{j}}, t\right)\right| \leq C h^{2-\epsilon},
$$

where $C$ is independent of $t$ and $h$.

Proof. We note that the choices $\mu=1-\epsilon / 4$ and $\alpha=\epsilon / 4$ satisfy all required inequalities in Theorem A.1. Moreover, all the necessary error terms will converge to zero exponentially as $h \rightarrow 0$ with the exception of the error bound on $I_{a}$ given in equation (A.14). With the chosen $\mu$ and $\alpha$ this term satisfies

$$
I_{a} \leq C h^{2-\epsilon},
$$

proving the corollary.

Appendix B. Numerical Methods for Evaluating $p_{h}(\boldsymbol{x}, t)$ and $p_{h}^{(i)}(\boldsymbol{x}, t)$. All reported simulations were performed using MATLAB. The numerical calculations in both Subsections 2.1 and 2.2.3 rely on evaluation of $G_{h}(\boldsymbol{x}, t)$, the Green's function for the discrete-space continuous-time diffusion equation, given by equation (2.8). To rapidly, and accurately, evaluate this function we rewrite it as

$$
G_{h}(\boldsymbol{x}, t)=\prod_{k=1}^{3} g_{h}\left(x_{k}, t\right),
$$

where

$$
g_{h}\left(x_{k}, t\right)=2 \int_{0}^{1 / 2 h} e^{-4 D t \sin \left(\pi h \xi_{k}\right) / h^{2}} \cos \left(2 \pi x_{k} \xi_{k}\right) d \xi_{k} .
$$

For the numerical calculations in Subsection 2.2.3 we evaluated $g_{h}\left(x_{k}, t\right)$ using MATLAB's built-in adaptive Gauss-Lobato quadrature routine, quadl. This routine was found to be too slow for the repeated evaluations required in the calculations of Subsection 2.1. There we instead numerically evaluated $g_{h}\left(x_{k}, t\right)$ using the trapezoidal rule, after applying the double exponential transformation for a finite interval described in [35]. For similar absolute error tolerances this method was substantially faster than quadl.

In Subsection 2.1, $p_{h}(\mathbf{0}, t)$ was found using a Gregory method [13] to solve the Volterra equation of the second kind, equation (2.12). We found it necessary to use 


\begin{tabular}{|c|c|c|c|c|c|c|}
\hline$n^{\prime}$ & $0, n$ & $1, n-1$ & $2, n-2$ & $3, n-3$ & $4, n-4$ & $4<n^{\prime}<n-4$ \\
\hline$\omega_{n^{\prime}}$ & $\frac{95}{288}$ & $\frac{317}{240}$ & $\frac{23}{30}$ & $\frac{793}{720}$ & $\frac{157}{160}$ & 1 \\
\hline
\end{tabular}

Table B.1: Gregory method weights, $\omega_{n^{\prime}}$, for $n^{\prime}=0, \ldots, n$.

a sixth order method to resolve $p_{h}(\mathbf{0}, t)$ accurately with a computationally tractable number of time-points. For comparison, the fourth order Gregory method described in [13] would have required more time points than available memory on our computer system to achieve the desired absolute error tolerance.

The sixth order Gregory method we used is based on discretizing time, $t_{n}=n \Delta t$, and calculating an approximate solution, $u_{n}(\mathbf{0}) \approx p_{h}\left(\mathbf{0}, t_{n}\right)$. The discrete equations satisfied by $u_{n}(\mathbf{0})$ are

$$
u_{n}(\mathbf{0})=G_{h}\left(\boldsymbol{x}_{0}, t_{n}\right)-k \Delta t \sum_{n^{\prime}=0}^{n} G_{h}\left(\mathbf{0}, t_{n}-t_{n^{\prime}}\right) u_{n^{\prime}}(\mathbf{0}) \omega_{n^{\prime}}
$$

where the weights of the Gregory rule are given by Table B.1 To start this method we require values for $u_{0}(\mathbf{0}), u_{1}(\mathbf{0}), \ldots, u_{8}(\mathbf{0}) . u_{0}(\mathbf{0})$ is given by the initial condition

$$
u_{0}(\mathbf{0})=G_{h}\left(\boldsymbol{x}_{0}, 0\right)=0 .
$$

The other values were obtained by using a sixth order explicit Runge-Kutta method. (See [13] for details of using explicit Runge-Kutta methods to solve Volterra integral equations of the second kind, and [32] for the specific method we used).

If one naively solves (B.1) by advancing from one time to the next, using $u_{0}(\mathbf{0}), \ldots$, $u_{n-1}(\mathbf{0})$ to calculate $u_{n}(\mathbf{0})$, the total work in solving for $N$ time points will be $O\left(N^{2}\right)$. The discrete convolution structure of (B.1) can be exploited by the FFT-based method of [22] to reduce the total work to $O\left(N \log ^{2}(N)\right)$. In practice we required this optimization to solve (B.1) in a reasonable amount of time. An important technical point we found was that both MATLAB's built-in discrete convolution routine, conv, and the MATLAB Signaling Toolbox FFT-based method, fftfilt, performed poorly for sufficiently large vectors. Our final code used the convfft routine [41], which performed significantly faster for large vectors.

We found this solution method computationally effective for $h$ as small as $2^{-11}$. Below this mesh size we encountered stability problems with the Gregory discretization. Moreover, to obtain the same absolute error tolerances used for coarser mesh sizes, the simulations required more time points than could be stored in the four gigabytes of system memory on our workstation. We also tried several [36, 45] existing spectral methods for numerically solving Volterra integral equations of the second kind, but found that in practice they were unable to obtain comparable accuracies to the Gregory method described above in solving equation (2.12).

Once $u_{n}(\mathbf{0})$ was calculated, we solved for $u_{n}(\boldsymbol{x}) \approx p_{h}\left(\boldsymbol{x}, t_{n}\right)$ by discretizing equation (2.11) to give

$$
u_{n}(\boldsymbol{x})=G_{h}\left(\boldsymbol{x}-\boldsymbol{x}_{0}, t\right)-k \Delta t \sum_{n^{\prime}=0}^{n} G_{h}\left(\boldsymbol{x}, t_{n}-t_{n^{\prime}}\right) u_{n^{\prime}}(\mathbf{0}) \omega_{n^{\prime}},
$$

where $\omega_{n^{\prime}}$ is again defined by Table B.1. 
Finally, for the figures in Subsection 2.2.3 each value of $p_{h}^{(1)}(\boldsymbol{x}, t)$ was calculated using composite Simpson's rule. $p_{h}^{(2)}(\boldsymbol{x}, t)$ was calculated by reusing composite Simpson's rule on the calculated values of $p_{h}^{(1)}(\boldsymbol{x}, t)$.

\section{REFERENCES}

[1] M. Abramowitz and I. A. Stegun, Handbook of mathematical functions with formulas, graphs, and mathematical tables, Dover Publications, 1965.

[2] J. L. Adelman and S.S. Andrews, Intracellular pattern formation: A spatial stochastic model of bacterial division site selection proteins MinCDE, Proc. Santa Fe Inst. Summer School, 2004.

[3] S. Albeverio, Z. Brzeźniak, and L. Dąbrowski, Fundamental solution of the heat and Schrödinger equations with point interaction, J. Funct. Anal. 130 (1995), 220-254.

[4] S. Albeverio, F. Gesztesy, R. Høegh-Krohn, and H. Holden, Solvable models in quantum mechanics, 2nd ed., AMS Chelsea Publishing, 1988.

[5] S. Albeverio and P. Kurasov, Singular perturbations of differential operators, London Mathematical Society Lecture Note Series, no. 271, Cambridge University Press, New York, 2000.

[6] S. S. Andrews and D. Bray, Stochastic simulation of chemical reactions with spatial resolution and single molecule detail, Physical Biology 1 (2004), 137-151.

[7] A. Arkin and H. H. McAdams, Stochastic mechanisms in gene expression, Proc. Natl. Acad. Sci. USA 94 (1997), 814-819.

[8] A. Arkin, J. Ross, and H. H. McAdams, Stochastic kinetic analysis of developmental pathway bifurcation in phage $\lambda$-infected Escherichia coli cells, Genetics 149 (1998), 1633-1648.

[9] F. Baras and M. M. Mansour, Reaction-diffusion master equation: A comparison with microscopic simulations, Phys. Rev. E 54 (1996), no. 6, 6139-6147.

[10] F. Berezin and L. Faddeev, A remark on Schrödinger equation with a singular potential, Soviet Math. Dokl. 2 (1961), 372-375.

[11] U. S. Bhalla, Signaling in small subcellular volumes. I. Stochastic and diffusion effects on individual pathways, Biophys. J. 87 (2004), no. 2, 733-744.

[12] W. J. Blake, M. Kaern, C. R. Cantor, and J. J. Collins, Noise in eukaryotic gene expression, Nature 422 (2003), 633-637.

[13] L. M. Delves and J. L. Mohamed, Computational methods for integral equations, Cambridge University Press, Cambridge, 1985.

[14] M. Doi, Second quantization representation for classical many-particle system, J. Phys. A: Math. Gen. 9 (1976), 1465-1477.

[15] J. Elf and M. Ehrenberg, Spontaneous separation of bi-stable biochemical systems into spatial domains of opposite phases, IEE Sys. Biol. 1 (2004), no. 2, 230-236.

[16] R. Erban, S. J. Chapman, and P. K. Maini, A practical guide to stochastic simulations of reaction-diffusion processes, Submitted, available at http://www2.maths.ox.ac.uk/ erban/papers/download.html.

[17] D. Fange and J. Elf, Noise induced min phenotypes in E. coli, PLoS Comput. Biol. 2 (2006), no. 6, 0637-0638.

[18] W. Feller, An introduction to probability theory and its applications, vol. II, John Wiley \& Sons, Inc., 1971.

[19] E. Fermi, Motion of neutrons in hydrogenous substances, Ricerca sci. 7 (1936), no. II, 13-52.

[20] C. W. Gardiner, Correlations in stochastic models of chemical reactions, J. Stat. Phys. 14 (1976), 307.

[21] D. T. Gillespie, Exact stochastic simulation of coupled chemical-reactions, J. Phys. Chem. 81 (1977), no. 25, 2340-2361.

[22] E. Hairer, CH. Lubich, and M. Schlichte, Fast numerical solution of nonlinear Volterra convolution equations, SIAM J. Sci. Stat. Comput. 6 (1985), no. 3, 531-541.

[23] M. Hazewinkel (ed.), Encyclopaedia of mathematics, ch. Integral transform, Springer-Verlag, 2002.

[24] K. Huang and C. N. Yang, Quantum-mechanical many-body problem with hard-sphere interaction, Phys. Rev. 105 (1957), no. 3, 767-775.

[25] S. A. Isaacson, Relationship between the reaction-diffusion master equation and particle tracking models, J. Phys. A: Math. Theor., Accepted.

[26] _ A stochastic reaction-diffusion method for studying the control of gene expression in eukaryotic cells, Ph.D. thesis, New York University, 2005. 
[27] S. A. Isaacson and D. Isaacson, The reaction-diffusion master equation, diffusion limited reactions, and singular potentials, To be Submitted.

[28] S. A. Isaacson and C. S. Peskin, Incorporating diffusion in complex geometries into stochastic chemical kinetics simulations, Siam J. Sci. Comput. 27 (2006), no. 1, 47-74.

[29] J. Keizer, Nonequilibrium statistical thermodynamics and the effect of diffusion on chemical reaction rates, J. Phys. Chem. 86 (1982), 5052-5067.

[30] F. Kühner, L. T. Costa, P. M. Bisch, S. Thalhammer, W. M. Heckl, and Gaub H. E., LexADNA bond strength by single molecule force spectroscopy, Biophysical Journal 87 (2004), 2683-2690.

[31] G. Lahav, N. Rosenfeld, A. Sigal, N. Geva-Zatorsky, A. J. Levine, M. B. Elowitz, and U. Alon, Dynamics of the p53-Mdm2 feedback loop in individual cells, Nat. Genet. 36 (2004), no. 2, $147-150$.

[32] H. A. Luther, An explicit sixth-order Runge-Kutta formula, Mathematics of Computation 22 (1968), no. 102, 434-436.

[33] H. Maamar, A. Raj, and D. Dubnau, Noise in gene expression determines cell fate in Bacillus Subtilis, Science 317 (2007), 526-529.

[34] D. A. McQuarrie, Stochastic approach to chemical kinetics, J. Appl. Prob. 4 (1967), 413-478.

[35] M. Mori and M. Sugihara, The double-exponential transformation in numerical analysis, J. Comput. Appl. Math. 127 (2001), 287-296.

[36] M. Muhammad, A. Nurmuhammad, M. Mori, and M. Sugihara, Numerical solution of integral equations by means of the sinc collocation method based on the double exponential transformation, J. Comput. Appl. Math. 177 (2005), 269-286.

[37] E. M. Ozbudak, M. Thattai, I. Kurtser, A. D. Grossman, and A. van Oudenaarden, Regulation of noise in the expression of a single gene, Nat. Genet. 31 (2002), 69-73.

[38] N. Pavin, H. Č. Paljetak, and V. Krstić, Min-protein oscillations in Escherichia coli with spontaneous formation of two-stranded filaments in a three-dimensional stochastic reactiondiffusion model, Physical Review E (Statistical, Nonlinear, and Soft Matter Physics) $\mathbf{7 3}$ (2006), no. 2, 021904.

[39] L. Peliti, Path integral approach to birth-death processes on a lattice, J. Physique 46 (1985), 1469-1483.

[40] J. M. Raser and E. K. O'Shea, Control of stochasticity in eukaryotic gene expression, Science 304 (2004), 1811-1814.

[41] L. Rosa, fftconv.m available at http://www.mathworks.com/matlabcentral/fileexchange.

[42] A. E. Smith, B. M. Slepchenko, J. C. Schaff, L. M. Loew, and I. G. Macara, Systems analysis of Ran transport, Science 295 (2002), 488-491.

[43] M. V. Smoluchowski, Mathematical theory of the kinetics of the coagulation of colloidal solutions, Z. Phys. Chem. 92 (1917), 129-168.

[44] G. M. Süel, J. G. Garcia-Ojalvo, L. M. Liberman, and M. B. Elowitz, An excitable gene regulatory circuit induces transient cellular differentiation, Nature 440 (2005), 545-550.

[45] T. Tang, X. Xu, and J. Cheng, On spectral methods for Volterra type integral equations and the convergence analysis, Submitted to J. Comput. Math.

[46] U. C. Täuber, M. Howard, and B. P. Vollmayr-Lee, Applications of field-theoretic renormalization group methods to reaction-diffusion problems, J. Phys. A: Math. Gen. 38 (2005), no. 17, R79-R131.

[47] J. S. van Zon, M. J. Morelli, S. Tănase-Nicola, and P. R. ten Wolde, Diffusion of transcription factors can drastically enhance the noise in gene expression, Biophys. J. 91 (2006), no. 12, $4350-4367$.

[48] J. S. van Zon and P. R. ten Wolde, Simulating biochemical networks at the particle level and in time and space: Green's function reaction dynamics, Physical Review Letters 94 (2005), no. $12,128103$. 